\title{
Sentinel Lymph Node Biopsy: Actual Topics
}

\author{
L.G. Porto Pinheiro' ${ }^{1}$ P.H.D. Vasques ${ }^{1}$, \\ M. Maia ${ }^{2}$, J.I.X. Rocha ${ }^{3}$ and D.S. Cruz ${ }^{3}$ \\ ${ }^{1}$ Department of Surgery, Faculty of Medicine, UFC, Fortaleza - Ceará, \\ ${ }^{2}$ Biochemistry, Laboratory for experimental surgery \\ Saul Goldenberg MD, Fortaleza - Ceará, \\ ${ }^{3}$ Faculty of Medicine, UFC, Fortaleza - Ceará, \\ Brazil
}

\section{Introduction}

The incidence of female breast cancer has increased $0.5 \%$ since the year 2000, as reported by the International Agency Research on Cancer. This number is greater in developing countries due to the increase in life expectancy and change in behavior resulting in increased exposure to risk factors.

Lymph node staging is an early event carried out during initial patient evaluation in developed countries. Some $20 \%$ to $30 \%$ of all cases are diagnosed quite early (in situ lesions) DCIS; $79 \%$ of the patients diagnosed at stage I and II has negative axillary nodes; (Holland et al., 2001). These values are somewhat different in developing countries where more than $50 \%$ of the cases are diagnosed at advanced stages of the disease.

Increased survival rates have been reported lately. According to Parkin (Parkin et al., 2001), the overall survival rate is $91 \%$ after the first year and $65 \%$ after five years in Europe. These values increase to $96.8 \%$ after the first year in the United States. As primary prevention of breast cancer is not available as yet, early detection and treatment in the initial phase of the disease are, therefore, the most important measures for its control (INCA, 2009). Available diagnostic methods include mammography, breast ultrasonography and fine-needle aspiration biopsy among others (Moore et al., 1996; Morton et al., 1998).

Until 1990, the assessment of axillary status of a patient with breast cancer depended on the histopathologic examination of lymph nodes from complete axillary dissection. With the definition of the sentinel lymph node as the first one which receives the drainage of the tumoral area (Cabanas, 1977), it was possible to ensure means for the proper staging of the illness and the therapeutic approach establishing less invasive surgery techniques.

Currently, it is admitted that the presence of metastatic lymph nodes is the main predictor factor for prognosis of the breast malignant neoplasia course and subsequent therapeutic program. Lymph nodes are also valuable for staging breast cancer. In the recent past dissection of the axillary lymph nodes was required for this matter, resulting in a series of additional complications (Veronesi et al., 1999). 


\section{Sentinel lymph node biopsy on breast cancer surgery}

The status of the axillary lymph nodes is one of the most important prognostic factors in women with early stage breast cancer. Histologic examination of lymph nodes is the most accurate method for assessing spread of disease to these nodes.

Axillary lymph node dissection (ALND) has traditionally been a routine component of the management of early breast cancer. The benefits of ALND include its impact on disease control (ie, axillary recurrence and survival), its prognostic value, and its role in treatment selection. However, the anatomic disruption caused by ALND may result in lymphedema, nerve injury, and shoulder dysfunction, which compromise functionality and quality of life.

ALND remains the standard approach for women who have clinically palpable axillary nodes or positive nodes confirmed by methods such as ultrasound guided fine needle aspiration. For patients who have clinically negative axillary lymph nodes, sentinel lymph node biopsy (SLNB) is a less morbid method of staging the axilla than ALND.

Indications for and outcomes of SLNB will be reviewed here.

The sentinel lymph node (SLN) technique is based upon the observation that tumor cells migrating from a primary tumor metastasize to one or a few lymph nodes (LNs) before involving other LNs. Injection of vital blue dye and/or radiolabeled colloid around the area of the tumor permits identification of a SLN in the majority of patients, and its status accurately predicts the status of the remaining regional LNs.

In patients with clinically node negative breast cancer, SLNB identifies patients without axillary node involvement, thereby obviating the need for more extensive surgery. Several studies have shown that the risk of arm morbidity, particularly lymphedema, sensory loss, and shoulder abduction deficits, is significantly less for SLNB than with standard axillary dissection. As an example, the risk of lymphedema after 12 months was reported as 2 percent after SLNB alone as compared with 13 percent after SLNB with axillary lymph node dissection (ALND) in the American College of Surgeons Oncology Group (ACOSOG) Z0011 trial.

Most surgeons and major cancer centers have adopted SLNB as a standard means of axillary nodal assessment. In a study of over 490,000 women with early breast cancer from the National Cancer Database, use of SLNB increased from 27 to 66 percent between 1998 and 2005 in the United States. Similar trends have been reported from Canada and the United Kingdom. SLNB is endorsed as an alternative to ALND for the diagnosis of axillary metastases in patients with clinically node-negative early breast cancer in guidelines from the American Society of Clinical Oncology (ASCO), the International Expert Consensus Panel on the Primary Therapy of Early Breast Cancer, and others.

Despite variability in selection criteria and technique, a SLN is consistently identified in approximately 96 percent of cases, and predicts the status of the remaining axillary LNs in $\geq 95$ percent of cases in most series. The false negative rate of SLNB was originally reported as 5 to 10 percent (sensitivity 90 to 95 percent), but lower rates are attainable by experienced surgeons.

The greatest concern with SLNB is the potential of a false negative result, which could increase the potential for axillary recurrence. However, despite the approximately 5 to 10 percent false negative rate with SLNB found in studies in which completion ALND has been done, several series suggest that axillary recurrence rates are low after a negative SLNB alone in early stage breast cancer (range 0 to 4.5 percent). The details of key trials validating the SLNB are described below: 
A landmark multicenter study of 443 patients with early breast cancer demonstrated that the SLNB technique could be learned and successfully applied by a diverse group of surgeons spanning private and academic practice. All patients underwent SLNB using radiolabeled colloid followed by completion ALND. At least one SLN was identified in 98 percent of cases and the predictive value of a negative SLN was 96 percent, with a false negative rate of 11 percent (sensitivity 88 percent). More intensive pathologic evaluation of the nodes in false negative cases with deeper sectioning of the sentinel node and immunohistochemical staining increased the yield of occult metastases in 18 percent of cases.

A systematic review, performed by the ASCO expert guidelines panel, included 69 eligible trials of SLNB in early stage breast cancer, representing 8059 patients. The SLN was identified using radiocolloid, blue dye, or both. SLN identification was successful in 95 percent of patients. The false negative rate was 7.3 percent (range 0 to 29 percent). The combination of radiocolloid and blue dye resulted in a significantly higher success rate in SLN mapping with a lower false negative rate as compared to blue dye alone.

The NSABP B-32 trial, published after the systematic review, enrolled 5611 patients with clinically negative nodes and compared SLNB followed by ALND versus SLNB followed by ALND only if the SLN was positive. Lymphatic mapping was successful in 97 percent, and the false-negative rate was 9.8 percent. No significant differences were observed in regional control, overall survival, or disease free survival between the groups at a median follow-up of almost eight years.

SLNB should be performed in most women with clinically node negative invasive or microinvasive breast cancer. SLNB can be omitted if the nodal information will not affect adjuvant treatment decisions. As an example, women $\geq 70$ years of age who have a small $(<2$ $\mathrm{cm}$ ) estrogen receptor-positive tumor and a clinically uninvolved axilla may be treated without a SLNB. Although older patients do not appear to be at increased risk of complications following axillary clearance, retrospective analyses and one randomised trial have questioned its value in older women with breast cancer (IBCSG, 1996). In a randomised trial, 473 women aged 60 or over. Interestingly in the ALMANAC trial (comparing sentinel node biopsy and standard axillary treatment), which used validated measures of patient reported quality of life and arm morbidity, older women (65 years and over) irrespective of axillary management had better quality of life outcomes at all stages of the 18-month followup than younger women (Fleissig et al, 2006).

SLNB should be performed in women with extensive ductal carcinoma in situ (DCIS), who are undergoing mastectomy. A SLNB will not be possible after mastectomy if invasive disease is found on final pathology, necessitating an axillary dissection for staging purposes. (See 'Ductal carcinoma in situ' below.)

When a SLNB is not successful or when clinically suspicious nodes are present in the axilla after all sentinel lymph nodes have been removed, the surgeon should perform an axillary dissection for staging purposes and to ensure locoregional control.

For women with clinically suspicious lymph nodes, preoperative axillary ultrasound (US) with fine needle aspiration (FNA) or core biopsy of suspicious areas provides a means to identify patients who have positive nodes, and thus need axillary lymph node dissection (ALND) rather than a SLNB. As an example, in a series of 653 consecutive patients, the preoperative diagnosis rate of axillary disease was 23 percent using axillary US and FNA, thereby avoiding the need for a second operation in 150 women. The efficacy of this approach is somewhat variable between centers because the accuracy of US examination is operator dependent. 
Approximately 40 percent of patients with a positive sentinel lymph node (SLN) will be found to have residual disease in the axilla SLN metastases are categorized as isolated tumor cells, micrometastases or macrometastases, depending on the size of the largest tumor deposit in the SN. Treatment options will depend on the clinical situation and include completion axillary lymph node dissection (ALND) or axillary radiation therapy (RT).

Isolated tumor cells - The seventh edition of the American Joint Committee on Cancer (AJCC) tumor node metastasis (TNM) staging system for breast cancer includes a stringent classification for lymph node findings of isolated tumor cell clusters and single cells. Small clusters of cells not greater than $0.2 \mathrm{~mm}$, or nonconfluent or nearly confluent clusters of cells not exceeding 200 cells in a single histologic lymph node cross section are classified as isolated tumor cells and are considered node negative. Malignant cells in regional lymph node(s) no greater than $0.2 \mathrm{~mm}$ (detected by H\&E or IHC including ITC) are designated as $\mathrm{pN} 0(\mathrm{i}+)$. Isolated tumor cells are not considered an indication for further axillary surgery, radiation treatment or adjuvant systemic therapy.

SLNB allows the pathologist to perform a more detailed study of one or a few LNs that are most likely to contain metastases, compared to the 15 to 25 LNs obtained with ALND. This has the potential to improve staging accuracy, but has led to an increase in the identification of micrometastatic nodal involvement. For this reason, there is a separate designation of pN1mi $(>0.2 \mathrm{~mm}$ and no greater than $2.0 \mathrm{~mm}$ ) to indicate micrometastases alone.

Although it seems intuitive that the finding of axillary micrometastases should worsen prognosis, most studies show no reduction in patient survival compared to those without micrometastases. However, some analyses do suggest a negative impact of micrometastases on breast cancer outcomes.

There is debate about the prognostic value of the size of the SLN micrometastases $(\leq 0.2 \mathrm{~mm}$ versus larger) in predicting the likelihood of involvement of axillary non-SLNs. Guidelines from ASCO and NCCN recommend that routine completion ALND be carried out for micrometastases detected on SLNB with standard hematoxylin and eosin (H\&E) examination. However, the indications for a completion ALND for micrometastases are the subject of controversy.

The 2005 American Society of Clinical Oncology (ASCO) guidelines and 2010 National Comprehensive Cancer Network $(\mathrm{NCCN})$ guidelines recommend that routine completion ALND be carried out for patients with SLNB macrometastases ( $\geq 2 \mathrm{~mm}$ ). However, the indications for a completion ALND in patients with $<3$ positive sentinel nodes is the subject of controversy.

Role of IHC and RT-PCR in sentinel node evaluation - Occult micrometastases refers to nodal metastases that are not seen on hematoxylin and eosin (H\&E) examination but are detected only by immunohistochemistry (IHC) or reverse transcriptase polymerase chain reaction (RT-PCR). The significance of occult micrometastases in terms of surgical management and patient outcome appears to be negligible.

Preliminary results from the American College of Surgeons Oncology Group (ACOSOG) study Z0010, a prospective multicenter study of 5210 patients with almost eight year follow up, confirm that IHC-detected metastases have no significant impact on overall survival. Thus, routine IHC or PCR is not recommended for the evaluation of SLNs in guidelines published by ASCO, NCCN, and others. Histologically negative nodes that are IHC or RT$\mathrm{PCR}$-positive are classified as $\mathrm{pN} 0$ disease in the TNM staging system for breast cancer. 
Role of IHC in invasive lobular carcinoma - Although routine IHC staining with cytokeratin is not indicated for most breast cancers, it can be helpful for examination of the sentinel nodes in patients with invasive lobular carcinoma since the morphology of lobular cancer can be difficult to detect on H\&E of axillary lymph nodes. In general, IHC should be used to definitively diagnose an area that is suspicious for, but not diagnostic of, lymph node metastases on H\&E rather than as a routine method of evaluating nodes in cases of invasive lobular cancer.

When should completion axillary dissection be performed? The need for completion axillary lymph node dissection (ALND) is dependent upon the SLNB findings. There are some clear indications and some settings in which optimal surgical approach is controversial.

There is general acceptance for the following approaches:

For patients with a negative sentinel lymph node biopsy (SLNB), completion ALND is not indicated.

Patients with SLNB showing isolated tumor cells only are considered node negative and completion ALND is not indicated.

For patients with a positive SLNB showing micrometastases or macrometastases in three or more nodes, detected with standard hematoxylin and eosin (H\&E) examination, completion ALND is recommended for staging purposes and to ensure local control. The timing of the procedure (ie, immediate [one operation] versus delayed [two separate operations]) does not seem to impact the total lymph node yield or the rate of long-term complications (particularly lymphedema).

In contrast, the need for a completion ALND is controversial in patients with a positive SLNB showing micrometastases or macrometastases in less than three nodes, detected with standard hematoxylin and eosin (H\&E) examination. The SLN is the sole tumor-bearing node in up to 60 percent of cases overall, and in almost 90 percent of patients who harbor only micrometastatic disease. These observations have led to speculation that completion ALND may not be necessary in selected patients with a positive SLNB in less than three nodes because the need for systemic therapy is established and the risk of an axillary recurrence appears to be low.

The ACOSOG Z-0011 trial was designed to address the need for completion ALND for patients with T1 or T2 tumors that were clinically node negative and had less than three positive sentinel nodes; all patients were treated with radiation to the breast. Target accrual was 1900 patients. The study closed prematurely because of low accrual and low event rate after enrolling 425 patients in the SLNB alone arm and 388 in the SLNB plus ALND. The majority of patients had estrogen receptor positive tumors. At a median follow-up of 6.3 years, there were no significant differences between the SLNB plus ALND group versus the SLNB alone group in the locoregional recurrence rate (in breast recurrence 3.7 versus 2.1 percent; nodal recurrence 0.6 versus 1.3 percent), overall survival ( 91.9 versus 92.5 percent) or disease free survival (82.2 versus 83.8 percent). Estrogen receptor status and adjuvant systemic therapy were independent predictors of survival.

Based upon the apparent lack of benefit and low risk of events in this trial, some have suggested that completion ALND is not necessary for women with T1 or T2 tumors that are clinically node negative with less than three positive SLNs who will be treated with whole breast radiation, particularly in women with estrogen receptor positive tumors. Others prefer to wait for results from two ongoing randomized trials studying the benefit of ALND for clinically node negative women with positive SLNs: the EORTC 10981-22023 AMAROS trial; and Trial 23-01 of the International Breast Cancer Study Group (IBCSG). 
Until more data are available, the need for completion ALND in clinically node negative women with less than three positive SLNs who will be treated with radiation to the breast is best resolved on a case-by-case basis, taking into account the patient's other risk factors and comorbidities and patient preference. When completion ALND is omitted in patients with a positive SLNB, radiotherapy is recommended.

Predictive nomograms - SLNB followed by a completion ALND results in significantly greater arm morbidity than SLNB alone. Because the majority of patients with a SLNB metastases will not have additional positive nodes on completion ALND, several predictive nomograms for estimating the risk of additional positive nodes have been developed in an effort to spare women from unnecessary and potentially morbid surgery. These nomograms include both clinical and pathologic features, such as the size and/or number of the SLN metastases, extranodal extension, and the size and/or presence of lymphovascular invasion in the primary tumor. A retrospective analysis of 319 patients with a positive SLNB who underwent completion axillary dissection compared the performance of four different nomograms. None of the nomograms was sufficiently reliable for clinical use.

Sentinel lymph node biopsy (SLNB) remains controversial in selected clinical settings.

Male breast cancer - The vast majority of published studies of SLNB for breast cancer are in women. Data are limited in men with breast cancer (MBC), because it is uncommon. A retrospective study of 30 men with breast cancer reported a 100 percent SLNB identification rate and a false negative rate of 0 percent. Prospective studies establishing the sensitivity and specificity of SLNB in MBC have not been carried out. However, the principles guiding SLNB in women appear to apply to men.

Due to the limited amount of data, the 2005 American Society of Clinical Oncology (ASCO) guidelines on SLNB did not make a specific recommendation about the use of SLNB in $\mathrm{MBC}$, although it was deemed "acceptable".

Most studies have restricted SLNB to T1 or T2 breast cancers $<5 \mathrm{~cm}$ in size, since larger tumors have a higher likelihood of positive axillary nodes. However, some studies have shown that SLNB is accurate in patients with T3 tumors and clinically negative axillae. Thus, many clinicians do not recognize large breast tumors as a contraindication to SLN dissection, as long as the axilla is clinically negative.

However, patients with T4 tumors (locally advanced) or inflammatory breast cancer are not considered candidates for SLNB. The false negative rate is high in patients with inflammatory breast cancer, presumably because of the presence of partially obstructed, functionally abnormal subdermal lymphatics.

The 2005 ASCO guidelines on SLNB did not recommend the routine use of SLNB in patients with locally advanced or inflammatory breast cancer for whom ALND was recommended to ensure locoregional control. Consensus recommendations from an International Expert Panel published in 2010 considered inflammatory breast cancer to be the one of the few absolute contraindications to SLNB. In addition, SLNB was not recommended for T4 tumors.

Many women with large primary breast tumors are offered neoadjuvant chemotherapy prior to definitive locoregional therapy. The optimal timing for sentinel lymph node biopsy (SLNB) in patients receiving neoadjuvant therapy has been debated, because some have reported a higher false negative rate for SLNB performed after induction therapy. Other uncontrolled studies support the accuracy of SLNB in such patients.

The 2005 ASCO guidelines concluded that there was insufficient information to guide the appropriate timing for SLNB in patients receiving preoperative systemic therapy. However, 
if prognostic information gained from examination of the axillary nodes is deemed valuable for planning locoregional treatment, SLNB can be considered before the institution of systemic therapy. Completion axillary dissection, if indicated, can be performed following chemotherapy at the time of definitive surgery. The ACOSOG trial Z1071, which is still accruing patients, is designed to answer the question of SLNB accuracy after neoadjuvant chemotherapy.

Studies that evaluated the functional anatomy of lymphatic drainage support the theory that all quadrants of the breast drain into the same lymph node(s). Thus, subareolar and intradermal (rather than peritumoral) injection of radiolabeled colloid or blue dye render SLNB feasible for patients with multicentric disease.

The success of SLNB for multicentric disease has been demonstrated in several studies. In a study of 142 women with multicentric breast cancer, SLNB was successful in 91 percent, with a false negative rate of 4 percent. However, the number of patients requiring completion ALND because of a positive SLN is higher in multicentric compared with unicentric disease. The likelihood of finding additional disease at the time of completion ALND is also higher with multicentric disease. The ASCO guidelines recommend SLNB as appropriate for patients with multicentric disease.

Most women with ductal carcinoma in situ (DCIS) do not require assessment of the axillary nodes, particularly if they are undergoing breast conserving therapy. However, women with DCIS may be candidates for SLN mapping if they are undergoing mastectomy, because the performance of SLNB will be impossible at a later time if invasive disease is found. An intact breast with its lymphatic plexus is necessary for injection of both the blue dye and the radioisotope tracers.

Some recommend that SLNB be considered in patients who are undergoing breast conserving therapy or mastectomy for DCIS only if the risk of node metastases is increased, as with extensive high-grade DCIS, a strong suspicion of invasive disease based upon ancillary imaging, or documented microinvasive disease in the core biopsy. However, if a lumpectomy is performed and invasive disease is identified, a SLNB can be done as a separate operation. This approach can minimize unnecessary morbidity, since SLNB can be associated with complications. SLNB is generally performed if microinvasion is found in the breast biopsy or if mastectomy is required for extensive or multifocal DCIS, where the risk of an occult invasive component is increased.

The finding of isolated tumor cell clusters (ie, pNO(i+)) in SLNs from patients with DCIS changes the clinical disease stage to stage IB in the seventh edition of the TNM staging system.

The safety and test performance of SLNB during pregnancy has not been fully evaluated. Supravital dyes such as isosulfan blue dye should not be administered to pregnant women. Available data suggests that the dose of radiation to the fetus is minimal using radiocolloid during SLNB. Nonetheless, 2005 guidelines from ASCO recommend against the use of SLNB in pregnant women with early stage breast cancer.

The feasibility of SLNB in women who have undergone other non-oncologic types of breast surgery such as reduction mammoplasty or augmentation with breast implants is unclear. The expert panel convened by ASCO did not make a recommendation for or against SLNB in women who have had breast reduction or augmentation because of insufficient data. They suggested that if SLNB were considered in this setting that it might best be performed with preoperative lymphoscintigraphy. 
SLNB after axillary surgery has not been widely studied. In one retrospective series, a SLN could not be identified in 25 percent of 32 cases in which SLNB was attempted in women who had undergone prior axillary surgery. Guidelines from ASCO recommend against SLNB in women who have undergone prior axillary surgery. However, there are accumulating reports of successful second SLNB in patients with a local breast cancer recurrence following a previous SLNB and/or axillary dissection. This practice is becoming more frequently employed and further study is indicated, including the optimal interval before repeat sentinel node biopsy should be attempted. Lymphoscintigraphy should be performed if repeat sentinel node biopsy is planned, given that these patients often have alternate drainage patterns.

SLN techniques can identify non-axillary metastases in up to 43 percent of cases, depending upon the volume and type of colloid injected, injection technique, and primary tumor location and size. Whether or not this is useful remains controversial, since the majority of the data regarding treatment decisions and outcomes comes from evaluation of only axillary nodes.

Positive IM nodes are most common with medial tumors over $2 \mathrm{~cm}$ in size. The clinical relevance of finding and treating IM nodal disease in breast cancer is highly controversial.

Patients with multiple positive axillary nodes usually have the IM node chain included in the radiation field, if it can be safely undertaken without a significant dose to the heart. However, it remains unclear whether inclusion of the IM nodes in the treatment field is responsible for the survival benefit seen with chest wall radiation. Definitive demonstration of benefit for the treatment of IM nodes in the radiation field requires a clinical trial in which women are randomly assigned to IM-directed RT fields or not. Two such trials have completed accrual, and results are forthcoming: one sponsored by the EORTC (protocol 22922) and the other by the National Cancer Institute of Canada (protocol MA 20).

Patients with axillary node-negative disease will be found to have regional metastases to the IM nodes in 8 to 10 percent of cases. The diagnosis of positive IM nodes might benefit those patients who would otherwise not be candidates for adjuvant systemic therapy. Some have suggested that patients with primary IM nodal drainage on lymphoscintigraphy receive radiation treatment targeting the IM chain. However, many surgeons do not employ radiotracer injection and use only an intraoperative injection of blue dye to identify the sentinel nodes. Additional noninvasive methods for IM node assessment may be helpful, including MRI or PET scanning, although these imaging modalities are unable to definitively identify positive nodes.

There are also limitations to the SLN technique for identification of IM nodes. SLNB does not reliably identify involved IM LNs because of interference from radioactivity at the primary tumor site. There is a high rate of technical failure (20 to 39 percent) in patients with parasternal hot spots on lymphoscintigraphy. In addition, not all hot spots in the IM region represent tumor involvement.

In addition, the IM nodes are difficult to sample surgically if a positive hot spot is identified in this area. Although IM biopsy can be accomplished at the time of mastectomy by splitting the fibers of the pectoralis major, an IM node biopsy in a patient undergoing breast conserving surgery usually requires a second incision, which is cosmetically visible in many types of clothing. The procedure can be complicated by pneumothorax, pleural effusion or bleeding.

Not surprisingly, there is disagreement among major groups on the issue of surgical management of the IM nodes. There is no consensus on the need for IM nodal dissection in 
women with detection of an IM SLN. Internal mammary nodes are not routinely dissected in patients undergoing breast conserving therapy or mastectomy with axillary lymph node dissection. Thus, in the absence of definitive data, dissection of the IM nodes with sentinel lymph node biopsy should still be considered investigational.

Numerous case reports document identification of intramammary lymph nodes on SLNB, although few have explored the clinical significance of this finding. Intramammary LNs are present in 1 to 28 percent of women with breast cancer. Most series report a high likelihood of additional axillary nodal metastases when the intramammary nodes contain cancer.

If intramammary nodes contain tumor, they have the same prognostic significance as a positive axillary LN with respect to staging. ALND should be considered for women with a positive intramammary LN on SLNB, even if the axilla is clinically negative, because of the high rate of axillary LN involvement in these women.

Many studies have sought to determine the optimal technique for SLNB. Using a combination of isotope and blue dye for sentinel node localisation drastically reduces the rates of failed and false-negative procedures. In the ALMANAC study, in most patients both dye and isotope found the sentinel nodes, however, in approximately $10 \%$ of patients only one of the agents located the sentinel node. More importantly, in approximately $4 \%$ of patients the positive SLN was found by dye alone and in 3\% by isotope alone; these would have been missed by relying on a single technique of localisation.

They have shown previously that replacement of the SLN by tumor significantly reduces the radioisotope uptake and may adversely affect intra-operative SLN identification. However, SLN identification using blue dye is not compromised by increased SLN tumor burden. The afferent lymphatic leading to the blocked node may be patent. The surgeon can identify the tumor-replaced node by following the blue lymphatic leading to the node. This result further suggests that a combination of blue dye and radioisotope should be used to optimise the localisation rate.

The ALMANAC study found that patient age, BMI, sentinel node visualisation on preoperative lymphoscintiscan, tumor location, tumor size, tumor histology and presence of multifocality did not alter the false-negative rate. They have shown that high BMI adversely influences the successful mapping of SLNs. Patient age did not alter SLN localisation, though it has been reported in several other studies which have shown that accurate identification of the SLN decreases with increasing age as well as weight. The specific causes for mapping failure in overweight patients are unclear. Sentinel node identification may be difficult in obese women because of the higher content of subcutaneous and axillary adipose tissue. Furthermore, the increased fatty tissue may impede the flow of the tracer through the lymphatics in the breasts of these patients. Or the lymph nodes in obese patients may have undergone fatty degeneration reducing their capacity to concentrate the tracer. These findings, however, do not contraindicate SLNB in obese individuals as the rate of successful localisation remains high and unsuccessful mapping does not adversely affect their prognosis or treatment.

Arguments have been made in favour of pre-operative lymphoscintigraphy as a road map for surgeons. The ALMANAC results indicate that SLN visualisation on preoperative lymphoscintigraphy significantly improves the intra-operative SLN identification rate $(\mathrm{p}<0.001)$. If an SLN takes up enough radiocolloid to image with a camera, it should be easily detected with the intraoperative probe. Similar findings were reported by Birdwell et al. 
They also found that tumors located in the upper outer quadrant had a higher SLN identification rate compared to other tumor locations. The simplest explanation relates to the transit distance for the blue dye or radioisotope from the peritumoral injection site to the axilla.

High false-negative rates may have a direct adverse impact on patient care including accurate staging, treatment decision making and long-term outcomes including survival. Clearly, the potential for both local as well as systemic under-treatment of patients increases as the false-negative rate increases. The ALMANAC study demonstrates a reduction in the predictive value of a negative SLNB in grade 3 tumors. Grade 3 tumors have a higher incidence of nodal metastases, thereby have an increased risk of lymphatic obstruction and re-routing of tracer leading to a false-negative result. Therefore, caution is required when applying the SLNB procedure in patients at considerably increased risk for lymph node positive disease.

The data from this study suggest that surgeons should not stop after finding just one SLN but should search thoroughly to be certain there are not more. This is important as the falsenegative rate in patients who had multiple sentinel nodes ( 3 or more) removed was $1.1 \%$ compared with $10.1 \%$ in those with 1 sentinel node removed.

Then, sentinel node biopsy is a safe and accurate alternative to routine axillary dissection for patients with early stage breast cancer with clinically negative axillary nodes. The success and accuracy of sentinel node mapping in breast cancer is optimised by the combined use of blue dye and isotope. Body mass index $>30$, tumor location other than upper outer quadrant and non-visualisation of SLN on pre-operative lymphoscintiscan adversely affect the SLN identification rate. SLNB may not be appropriate for patients who have grade 3 tumors, and the decision to perform the procedure should be determined on the basis of the clinical judgement of the treating surgeon.

\section{Hypersensitivity reactions and blue dye}

Hypersensitivity reactions to anesthetics and associated agents used during the perioperative period have been reported with increasing frequency in most developed countries. Their estimated incidence ranges between 1 in 5000 to 1 in 13,000 anesthetics (Mertes et al., 2002). Although neuromuscular blocking agents, latex, and antibiotics are the leading causative agents, every substance used during the perioperative period can be responsible for an immediate hypersensitivity reaction. (Mertes et al., 2003)

The combination of isotope and blue dye is the current recommended technique for sentinel node localization in breast cancer in many countries with localization rates of over $99 \%$. However, blue dye is associated with a range of allergic reactions. There have been a number of case reports and single case series of anaphylactic reactions following administration of patent blue V. Its allergic potential is also reflected in reports to the Committee of Safety of Medicines and to the manufacturer. No mortality has been reported but two cardio-respiratory arrests have been attributed to patent blue V (Mansel et el ., 2006; Montgomery et al., 2002).

Since the 1960s, blue dyes have been used in a variety of clinical situations, ranging from cardiac output determination (Al-Fadly et al., 1956) and lymphangiography (Biran et al., 1973) to intraoperative lymphatic mapping and sentinel lymphadenectomy in various types of 
neoplasms (Bilchik et al., 1998). They have long been considered a rare cause of anaphylaxis. This might in part be due to misleading nomenclature (Scherer et al., 2006). Patent BlueV (also called E131,Acid Blue 3, and disulfine blue) and isosulfan blue (also called Patent Blue violet or Lymphazurin), which belong to the group of triarylmethane dyes and share the same formula, are the most commonly used. A recent literature review that includes various names of these dyes reveals an impressive number of case reports of hypersensitivity reactions (Cimmino et al., 2001), and it has been suggested that sensitization occurs with everyday products containing blue dyes (Kalimo et al., 1981). In view of the increasing use of blue dyes for lymphatic mapping for sentinel lymph node biopsy, the incidence of anaphylaxis to these drugs can be expected to increase. Based on several retrospective and prospective studies, the estimated incidence of reactions of all grades of severity varies between $1 \%$ and $2 \%$, with severe reactions being observed in $0.2 \%$ to $1.1 \%$ of cases (Cox et al., 2000; Montgomery et al., 2002; King et al., 2004; Albo et al., 2003; Blessinget al., 2002). In a series of 1013 patients, preoperative prophylaxis with glucocorticoids, diphenhydramine, and famotidine was found to reduce the severity but not the overall incidence of adverse reactions to dye (King et al., 2004). Similarly, a nonsignificant trend toward fewer allergic reactions with smaller volumes of blue dye has been reported (Raut et al., 2006).

The clinical diagnosis of an immediate hypersensitivity reaction during anesthesia is generally considered difficult. Many signs can be misinterpreted as an interaction between the clinical status of the patient and the drugs administered a dose-related side effect of the drugs, or excessively light anesthesia. Vigilance is essential because reactions might be well established before they are detected. Therefore information concerning the various clinical features encountered is essential, particularly when reactions caused by injected dye are considered. Indeed, the clinical diagnosis of anaphylaxis is, in fact, presumptive based on a close temporal relation with the administration of the implicated substance. This is clearly not the case for hypersensitivity reactions elicited by dyes. In general, the clinical reactions respond to the definition of an "immediate" reaction occurring within less than 1 hour after exposure, they significantly differ from those caused by neuromuscular blocking agents or antibiotics, which usually occur less than 10 minutes after intravenous administration in a sensitized patient (Mertes et al., 2003).

We recall that allergic reactions are classified into four grades (Montgomery et al, 2002):

- grade I corresponds to the cutaneous signs generalized erythema, urticaria with or without edema angioedema;

- grade II is a multi-organ damage moderate with cutaneous signs, hypotension but greater than $70 \mathrm{~mm} \mathrm{Hg}$ and tachycardia unusual bronchial hyperreactivity (cough, difficulty breathing);

- $\quad$ grade III corresponds to a multiple organ damage severe threat, ant life and requiring special treatment; collapse, tachycardia or bradycardia, heartbeat, bronchospasm. Cutaneous signs may be absent or appear after the Remon-Wu blood pressure;

- $\quad$ grade IV corresponds to circulatory arrest and / or respiratory.

The mechanism underlying the allergic reaction to blue dyes remains unclear. Both direct mast cell activation, basophil activation, or both and cross-linking of specific IgE antibodies are possible causative factors (Woltsche-Kahr et al., 2000).

In conclusion, the use of Patent Blue $\mathrm{V}$ for localization of sentinel lymph nodes is associated with a significant number of hypersensitivity reactions. Many groups around the world are 
doing protocols to identify a patient that will be affected with hypersensitivity reaction after the use of blue dyes (Mansel et al., 2003). Maybe, with the development of new markers with less risk, we`ll find a solution for this.

\section{Sentinel lymph node biopsy: What's new?}

Cancer is known to develop via a multistep carcinogenesis process. Cancer treatments are performed on the basis of clinical and pathologic staging that is determined using morphologic diagnostic tools, such as conventional radiological and histopathological examinations (Pinheiro et al., 2000). Sentinel node localization is one the most important parameters considered in cancer diagnosis and therapy. The concept of the sentinel lymph node has earned widespread importance with the advent of the sentinel lymph node biopsy technique. This technique has been explored in staging of various types of cancers to determine their spread. Recent advances in molecular, biological, and genetic diagnostic techniques have led to a considerable exploration of the sentinel lymph node diagnostic agents and modalities and their implication for the involvement of Sentinel node in development and progression of cancer.

In the last decade, methods for the precise localization of sentinel lymph node (SLN) have drawn tremendous attention by cancer surgeons and researchers in the field of medical diagnosis. The accurate identification and characterization of lymph nodes by imaging has important therapeutic and prognostic significance in patients with newly diagnosed cancers. Amongst the various exploited methods for SLN diagnosis, nanocarriers have received increasing attention as lymph node delivery agents. The present review will focus on various such particulate carriers namely radiolabeled sulfur colloids, liposomes, quantum dots, dendrimers and magnetic nanoparticles, which are most extensively studied and have been attributed with the most desirable characteristics for SLN imaging (Ratnesh et al., 2009).

Nanotechnology, one of the recent leaps in technology progress, has provided a big boost to sentinel lymph node imaging. The most important advantage of nanocarriers in this regards is their ability to enable the targeted delivery of imaging agents for specific sentinel lymph node imaging. Accurate and quick identification of Sentinel node has now been rendered as an achievable target due to the advances in nanotechnology. For example, the use of Quantum dots (Chan et al., 2002), potentially allows rapid and accurate sentinel lymph node localization. sentinel lymph node imaging using several types of nanocarriers has been investigated and systematic investigation of these carriers is a need of the hour. Quantum dots have been used as fluorescent imaging agents for the in vivo imaging of sentinel lymph node. Conjugation of Quantum dots with biomolecules, including peptides and antibodies, has been used to target tumors in vivo. Quantum dots have also been employed to identify metastatic cancer cells, quantitatively measure the level of specific molecular targets and guide targeted cancer therapy by providing biodynamicmarkers for target inhibition.

Amongst the other nanocarriers explored, dendrimers have displayed long circulating positive contrast enhancement by MRI/ NIR imaging in various animal models (Bellin et al., 1998). These investigations suggest the possibility of utilizing a labeled MRI/NIR optical hybrid contrast agent containing nanocarrier for sentinel lymph node imaging. Recent animal studies with targeted nanobubbles (Dayton et al., 2001) for lymph node imaging and therapy have exhibited a significantly improved therapeutic efficacy of these carriers when 
compared with the nontargeted nanoparticles. This nanocarrier formulation offers the advantage of providing real-time reports of the treatment by means of ultrasound imaging since the nanocarrier contains both the imaging as well as the therapeutic agent.

The development of multifunctional nanoparticles may contribute significantly to the localization of sentinel lymph node in multiple types of cancer. Ideally, for constructing multifunctional nanoparticles, an appropriate combination of agents (imaging agent and targeting moiety) will have to be chosen, based on accurate biological information within the tumor and lymph node with imaging material attached on the nanoparticle surface (Forsberg et al., 2001). Nanoparticles may eventually be capable of detecting sentinel lymph node, pinpointing and visualizing their location in the body (real-time in vivo imaging). This advancement may eventually lead to complete elimination of cancerous cells identified by accurate sentinel lymph node localization. Several types of nanocarriers have been evaluated to identify their potential for sentinel lymph node imaging.

Despite all the new initiatives in the sentinel lymph node biopsy, it was never published a novel marker derived from blood for replacing the blue dye (Pinheiro et al., 2009), a member of the current gold standard with the technetium.

During the management of two patients submitted to breast biopsy in our service, we observed their skin mapped as the lymphatic ducts draining to axillary lymph nodes (Photo 1).

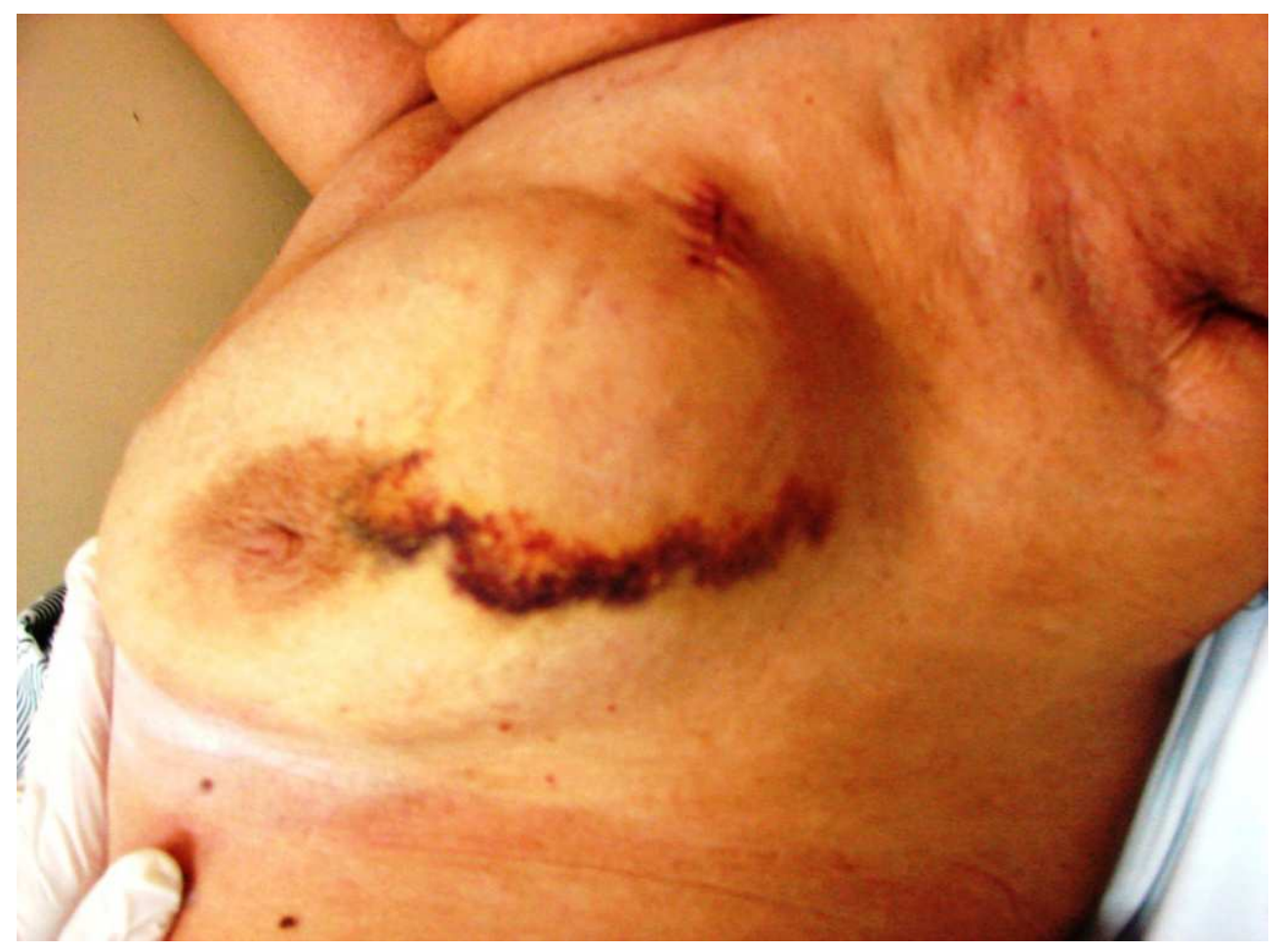

Photo 1. Skin mapped as a lymphatic duct in direction to the axilla 
This observation stimulated us to try an animal model which was described for our group. Our theory was that some blood derivate (hemosiderin) was captured by macrophages and accessed the lymphatic duct in direction to the axilla.

In bid to replicate what was seen in patients, we developed an experimental protocol for obtaining a marker derived from blood and tested in a model of experimental surgery in dogs (Photo 2).

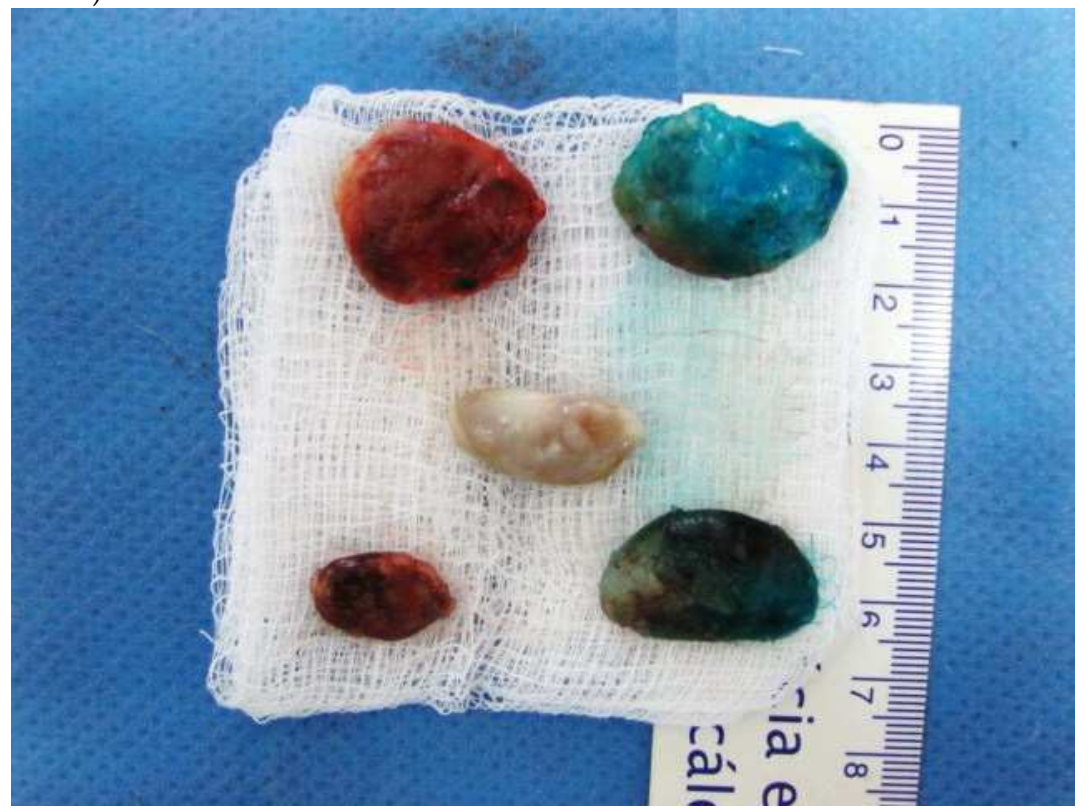

Photo 2. Sentinel lymph nodes marked with hemosiderin at left and with patent blue at right. Non-sentinel lymph node in the middle.

We found statistically significant and consistent data when comparing the group that underwent patent blue / technetium versus the group undergoing autologous bloodderived marker / technetium (Pinheiro et al., 2010).

The results of our experimental work showed that the blood derivate (hemosiderin) was capable of being used along with the radiomarker (technetium 99m) and coloring wonderfully the sentinel lymph node of female dogs.

The use of the blood derivate (hemosiderin) with the radiomarker also showed equal results comparing the use of the patent blue with the radiomarker in identifying the mammary sentinel lymph nodes of the female dog because they were always found in both axillas.

The hemosiderin, a blood-derived marker, theoretically does not cause side effects as allergic reactions. Besides, it may decrease personal and healthcare spendings, reduce the morbidity of the procedure and the emotional suffering, along with other indirect vantages.

\subsection{Lipossomes}

Liposomes have been used as delivery vehicles since the 1960s and their use for the delivery of imaging agents, for all imaging modalities, has a long history. Liposomes are defined as vesicles in which an aqueous volume is entirely surrounded by a phospholipid membrane. 
They can vary in size from $30 \mathrm{~nm}$ to several micrometers, and can be uni- or multilamellar. Their properties have been extensively investigated and can vary substantially with respect to their size, lipid composition, surface charge, and method of preparation.

Currently, liposomes are one of the most widely explored nanocarriers for delivering imaging agents to the SLNs. Many imaging agents can be covalently or non-covalently incorporated into the different compartments of these nanocarriers. Also, they can be designed to encapsulate a wide range of hydrophilic agents (dyes, drugs etc) within their aqueous interior. They also serve as promising lymphoscintigraphic agents since their surface can easily be modified by attaching or coating with specific receptors or other molecules such as polyethylene glycol (PEG) for selective targeting. The relative efficiency of entrapment of imaging materials into these nanocarriers has been established. Liposomes have also been used for cellular labeling of contrasting agents such as iron oxide nanoparticles to facilitate enhanced imaging. Literature also reports loading of liposomes with gamma-emitters and other radionuclides as well as specific receptor targeting ligands for application in various imaging modalities to facilitate diagnostic imaging of liver, spleen, brain, cardio-vascular system, tumors, inflammation and infection.

Liposomal carriers for lymph node drug delivery were first investigated by Segal et al., where an intratesticular injection of liposomes, encapsulating the anticancer drug actinomycin D, was carried out. Lymph node imaging using 99mTc-labeled liposomes was first performed by Obsorne et al. Although several clinical studies were performed soon after these initial attempts and reported promising results, the use of $99 \mathrm{mTc}$-liposomes for lymphoscintigraphy was questioned in an article by Patel et al. in 1984. In this report the stability of the $99 \mathrm{mTc}$-liposome complex as well as the integrity of the liposomes with in the lymph nodes was put under surveillance.

The early liposome involving imaging studies employed stannous chloride as a reducing agent to associate reduced $99 \mathrm{mTc}$ with the liposomal surface. This labeling method was subsequently shown to be unstable in vivo and lead to the development of more effective liposome labeling methods. Another major drawback which hampered the successful liposome targeting to the SLN was the lack of effective preoperative staining of regional lymph nodes to improve their intraoperative identification and thus the selectivity of 'sentinel node' lymphadenectomy. Unfortunately the most commonly used staining agent, blue dye, used in these early trials failed to provide the requisite intensity and duration of contrast, for enhanced imaging.

Since then several attempts have been made by researchers all over the world towards the development stable and effective liposome/99mTc-liposome based SLN imaging agents to tackle the afore-mentioned problems. One of these early development trials by Hirnle et al. utilized blue dye-encapsulated liposomes to localize the SLNs during surgery.

With this technique, it was studied that following the injection of the blue liposomes into the lymphatic vessels, the lymph nodes were stained blue. Most notably, retroperitoneal lymph nodes in rabbits remained dark blue up to 28 days after the hind limb endolymphatic instillation of liposomal blue dye. Promising results of these studies further paved a way to human trials, in which blue liposomes were injected directly into the lymphatic vessels of the foot of a patient prior to retroperitoneal staging-lymphadenectomy. Twenty four hours after the intralymphatic injection of the blue liposomes, when a surgery was performed, the lymph nodes were well stained with the blue dye and were readily visualized. 
These investigations proved the potential of liposomes as promising carriers for SLN imaging. However, the relatively low retention of liposomes in lymph nodes led to further investigations to improve this limitation.

\subsection{Dendrimers}

Dendrimers are perfectly cascade-branched, highly defined macromolecules, characterized by a combination of high end group functionality and compact molecular structures. The two major strategies of dendrimer synthesis in laboratories were initiated back in the year 1979.

The first method process included outward branching of an initiator core by a sequence of iterative reactions resulting in a three-dimensional organization of terminal groups. Fréchet et al. established the second process, known as the convergent growth process, which progressed inward from what would seem as a dendronmolecular surface to a reactive focal point at the root. The reaction of several, resulting reactive dendrons with a multifunctional core resulted in the formation of a dendrimer structure. The first dendrimer family, which was synthesized by structuring concentric shells of dendritic $\beta$-alanine units around a central initiator core, was the PAMAM (polyamidoamine) dendrimers.

These core-shell dendrimers grew linearly in diameter as additional shells/generations were added to their architecture. At the same time, at each generation, the surface groups underwent exponential amplification based on the dendritic-branching mathematics. Progression of this process over a few generations resulted in the formation of 'geometrically closed' nanostructure with guest-host container characteristics. Research over the years has resulted in the production and characterization of fifty such other dendrimer families, possessing interiors of varying compositions such as carbon, nitrogen, silicon, sulfur, phosphorus or metals. The architectural diversity of these nanostructures resulted in their application in biomedical functions including diagnostic imaging. Distinctive sizes, well defined structural design and the abundance of reactive and accessible peripheral functional groups were some of the features of this family of diagnostic vehicles which captured the interest of imaging experts. Other noteworthy features desirable for these applications included: structural control over size and shape of imagingagent cargo space; biocompatibility; non-toxicity of the polymer/pendant functionality employed; nanoscale-vehicles and/or scaffolding properties with high imaging-agent capacity; lack of immunogenicity; appropriate cellular adhesion; endocytosis and intracellular trafficking to allow imaging in the cytoplasm or nucleus; acceptable bioelimination or biodegradation; minimal nonspecific cellular and blood-protein binding properties; ease of consistent, reproducible, clinical grade synthesis.

In view of these valuable traits, several research groups have been actively engaged in designing dendrimers for diagnostic applications.

Dendrimers bearing GdIII complexes are being used as imaging agents in magnetic resonance imaging. Pioneering work in this area has been carried out byWeiner et al. This research group has reported dendrimer-based GdIII chelates consisting of polyamidoamine (PAMAM) dendrimers of generations 2 (G2) and 6 (G6), and possessing 12 and 192 reactive terminal amines, respectively, conjugated to the chelating ligand 2-(4-isothiocyanatobenzyl)6-methyldiethylenetria minepentaacetic acid (DTPA), through a thiourea linkage.

In vivo experiments on rabbits have revealed that intravenous injections of larger dendrimer conjugates provides enhanced circulation times of these structures of greater than $100 \mathrm{~min}$ 
thus resulting in excellent MRI images of the blood vessels. Another interesting area of research included the construction of DNA dendrimers which were synthesized by sequential hybridizations of partially complementary heteroduplexes (DNA monomers). After each generation, the structure was fixed by a crosslinking step. These DNA dendrimers were employed for high sensitivity detection of oligonucleotides since numerous radioactive or fluorescent labels could be tagged subsequent to the modification of the terminal polynucleotide strands. Such DNA dendrimers with capacity of selective recognition of specific RNA strands have been employed as selective markers of the Epstein-Barr virus in post transplant patients. Polynucleotide dendrimers labeled with fluorescent tags have also been proved as promising tools in DNA microarray technology for signal amplification in high-throughput functional genomic analysis.

Rapid diagnosis of genetic and pathogenetic diseases has been made possible by dendrimerbased biosensors for DNA hybridization. The single-stranded oligonucleotides immobilized on these structures specifically hybridize with their complementary target sequence. Also PAMAM dendrimers loaded with $111 \mathrm{In}$ or $153 \mathrm{Gd}$ complexes have been used as the anchoring vehicles for highly specific, radiolabeled monoclonal antibodies. In yet another study, the dendrimer arms were tagged with dye molecules which upon hybridization with a complementary fluorescently labeled DNA strand, generated energy transfer to provide enhanced imaging.

The first dual purpose dendrimers loaded with MRI-fluorescent imaging agents were described by Talanov et al. where PAMAM dendrimers were covalently attached to GdIIIDTPA chelates and units of the NIR fluorescent dye, Cy 5.5. In these systems, the bifunctional DTPA derivative, 2-(4-isothiocyanatobenzyl)-6-methyldiethylenetriaminepentaacetic acid (1B4M-DTPA) was chosen as the sequestering agent for GdIII , whereas the cyanine dye, Cy 5.5, was included to facilitate NIR imaging. These systems resulted in clear magnetic resonance and fluorescence images of the SLN(s). These dendrimers resulted in enhanced optical imaging, despite their powerful enhancement in the NIR, thus allowing the node localization through the skin. The blurred imaging by OI was taken care of by MRI which enabled the visualization of the node as well as its associated lymphatic channel. The superficial location of the node, in this study, resulted in the visualization of both the afferent and efferent lymphatic channels in the fluorescent image of the surgically exposed tissues. The research clearly demonstrates the immense potential of dendrimers as a platform for the development of efficient imaging agents for both magnetic resonance and optical imaging modalities.

Parallel work on the development of such dual mode dendrimers has also been reported by Koyama et al. where they have described the successful synthesis and utilization of dual labeled MRI/NIR optical hybrid contrast dendrimers for reoperative and intraoperative localization of SLNs. Yet another application of dendrimers in diagnostics is their application in dynamic MR lymphangiography. Gd-labeled dendrimer-based imaging agents have been used in mice models to detect the location of the SLN as well as to diagnose the presence or absence of metastatic cancer. Waldmann et al. have shown PAMAM-G6 dendrimers to be one of the best MRI imaging agents since they provide high concentrations of GdIII within the node, even in the presence of lymph node metastases.

These dendrimers exhibited most rapid and intense enhancement as compared to the other lymphatic agents tested. This utility of G6 dendrimers for lymphatic draining tumors opens up yet another avenue of their application for regional lymph nodes. However the widespread use of G6 dendrimers is limited due to certain drawbacks. 
For instance their application may be restricted in overt lymphatic metastases, where the metastases can obstruct lymphatic flow, leading to collateral lymphatic circulation, and thus prevent the dendrimers from entering into the SLN.

Another limitation is that since the GdIII concentrations within the lymph nodes are estimated based on both phantom studies and T1 measurements, it could not be accurately calculated based on relaxivity in the lymph node tissue. Since the tissue in the lymph node is not as homogenous as serum phantoms, this non-homogeneity was found to change the relaxivity of G6 and affect the T1 signal intensity due to susceptibility artifacts. However, since the values obtained with two different methods were nearly consistent, these MRI methods were deemed to provide a close estimation to the actual concentrations of GdIII within the lymph nodes, although the validation of this estimationwas found to be difficult in case of the lymph node specimens, which were too small to be resected and accurately weighed. The study thus revealed that the PAMAM-G6 Gd dendrimer not only provided excellent opacification of SLNs but also acted as potential carriers for targeted SLN therapy. Intensive research in the area of dendrimers as a potential tool for SLN imaging has thus revealed that certain pervasive biological patterns and biomimicry have played a distinctive role in the development of many biomedical applications for dendrimers. Research in this area has gained a huge momentum and around 3000 papers and patents about dendrimers as biomedical tools have been published in just last 5 years. The structural preciseness and control, reproducible and convenient size scale and comparable physico-chemical properties as that of globular proteins, antibodies and enzymes support the potential application of these tools in diagnostic imaging, particularly SLN imaging. This gamut of unique diagnostic properties offered by the dendrimers, along with their possible application in proteomics, clearly indicates their huge potential in the development of new biomedical devices and approaches for the detection and treatment of human diseases.

\subsection{Quantun dots}

Quantum dots (QDs) are nearly spherical semiconductor particles with diameters between 2-8 nm and containing roughly $200-10,000$ atoms. They are generally composed of atoms from group II and VI elements (e.g. CdSe and CdTe) or group III and V elements (e.g. InP and InAs) of the periodic table. The semiconducting nature and the size-dependent fluorescence of these nanocrystals have rendered them an extremely attractive approach for use in optoelectronic devices, biological detection, and also as fundamental prototypes for the study of colloids and the size-dependent properties of nanomaterials.

QDs provide a new class of biomarkers that could overcome the limitations of conventional diagnostics. Thus their application as luminescent imaging probes has significantly increased in the recent times.

When compared to the currently employed diagnostic techniques, QD based detection is rapid, easy and cost effective. The unique properties which make them ideal for use in diagnostics, include intense and stable fluorescence for a longer time; resistance to photobleaching, large molar extinction coefficients and highly sensitive detection due to their ability to absorb and emit light very efficiently. Due to their large surface area-tovolume ratio, a single QD can be conjugated to various molecules, thus making QDs appealing for employment in the fabrication of more complex and multifunctional nanostructures.

QDs have been covalently linked to a variety of biomolecules such as antibodies, peptides, nucleic acids and other ligands for fluorescence probing applications. Some of the 
applications of QDs in biology along with their tremendous potential for in vivo molecular imaging have already been explored. One of the greatest advantages which makes them suitable for imaging in living tissues is that their emission wavelengths can be tuned throughout the near-infrared spectrum by adjusting their composition and size, resulting in photostable fluorophores that are stable in biological buffers.

One of the first in vivo applications of quantum dots included mapping of the reticuloendothelial system (RES) and localization of SLNs. Fluorescence imaging provided a simple optical readout, a higher resolution as well as a wider dynamic range than dye absorption or scientigraphy techniques. For exposed tissues, the localization capability and sensitivity by QDs were found to be potentially better than those obtained by either visible dyes or radioactives, especially when using near-infrared emission. This could be attributed to the good tissue penetration and lowered background in the infrared offered by QDs.

Near-infrared (NIR) fluorescent QDs have been used for SLN mapping and resection. NIR light, otherwise invisible to the human eye, provides extremely high signal-to-background ratios without changing the look of the surgical field. A thorough discussion of the use of NIR light in biomedical imaging has been published previously.

With a suitable intraoperative imaging system, the NIR QDs provided several advantages for SLN mapping which included high sensitivity, real-time and simultaneous visualization of both surgical anatomy and lymphatic flow and non-radioactive detection. Kim et al. explored the possibility of employing NIR QDs emitting at $850 \mathrm{~nm}$ for SLN mapping. In this investigation, QDs injected intradermally into live mice were followed in real-time even up to $1 \mathrm{~cm}$ below the skin in the SLN. A series of 3D MR lymphangiograms recorded at $24 \mathrm{~min}$ after injecting G6 nano-size contrast agents. Possible accumulation of high concentrations of $\mathrm{Gd}(\mathrm{III})$ within the nodes as indicated by the phantom having 400 and 800 ppm of Gd(III) of the G6 agent.

In yet another study in pigs, the quantum dots were introduced into the lungs as a method of finding the SLN draining from lung cancer. The lymph node draining from the lung was rapidly identified. This rapid SLN identification using a non-radioactive method was thought to attribute a significant advantage to the method.

Various studies have been carried out for exploring the application of different materials that could be used in the fabrication of either the QD surface or core. Quantum dots with a core/shell/shell structure consisting of an alloyed core of InAsxP1-x, an intermediate shell of InP, and an outer shell of ZnSewere developed by Kimet al. Here, the cores contained a zinc blend structure for all compositions and exhibited a tunable emission in the NIR region. The first shell of InP was instrumental for bringing about a red-shift and subsequent increase in quantum yield, while the final shell of ZnSe stabilized the system for applications in aqueous environments, including NIR biomedical fluorescence imaging. This NIR-emitting core/shell/shell In AsxP1-x/InP/ZnSe was successfully used in SLN mapping experiments in various animal models.

In a recent study, PEG-coated quantum dots with terminal carboxyl, amino, or methoxyl groups have been used for SLN imaging. After injection, these quantum dots were shown to rapidly migrate to SLNs. At least two nodes could usually be defined when their passage from the tumor through lymphatics to adjacent nodes was visualized dynamically through the skin. Also, imaging studies performed during necropsy confirmed the confinement of quantum dots to the lymphatic system and thus proved them to be effective markers of SLN pathology. 
Several studies have been carried out to determine the in vivo fate of QDs. In one such investigation, within minutes of intradermal injection into female SKH-1 hairless mice, the highly UV fluorescent QDs could be observed moving from the injection sites, through the lymphatic duct system to the regional lymph nodes. These results indicated the potential of sentinel organs as effective locations for monitoring the transdermal penetration of these systems.

All these investigations have led to the emergence of QDs as technological conjectures with characteristics that could greatly improve SLN imaging and detection. However future concerted efforts are required in this promising area of research. Some of the important considerations which could be focused in future studies include: 1. Overcoming the possibility of toxicity from the heavy metal, particularly cadmium, which forms a major component of these systems. Toxicology investigations including their distribution, excretion, metabolism, pharmacokinetics, and pharmacodynamics would be imperative for the development of novel and more effective QDs for application in SLN imaging and beyond. 2. Combining quantum dot fluorescence imaging with other noninvasive techniques (e.g., scientigraphy, MRI, or PET) would allow mapping of deep nodes and more detailed information about SLN. 3. Quantum dots conjugated to appropriate ligands and fluorescing at different wavelengths could be explored to localize metastases in SLNs. 4. Development of biocompatible QDs with specificity may be explored to avoid their nonspecific organ uptake and RES scavenging. They could thus provide a better clinical option for effective imaging of SLN. 5. Optimizing the size, shape, and surface chemistry of QDs may lead to the development of novel QDs with enhanced and specific applications.

\subsection{Nanoparticles}

Nanoparticles have now been developed as widely used diagnostic agents in medicine. They possess unique features that can be exploited to suit the various imaging modalities. Many types of nanoparticles exhibit significant retention, sometimes as high as $40 \%$, in the first lymph node that they encounter during their movement from the injection site. This makes them ideal system for enhanced identification and localization of SLNs. Various studies had been reported for SLN imaging using different types of nanoparticles.

\subsubsection{Radiopharmaceutical colloidal nanoparticles}

Radiopharmaceutical colloidal preparations were developed soon after World War II. In 1955, Hultborn et al. reported the preoperative use of interstitial colloidal gold injections in breast cancer patients. With future investigations, $99 \mathrm{mTc}$ was introduced as a replacement for radioactive gold since it is readily available, inexpensive and has ideal imaging and dosimetry characteristics when compared to the latter. The standard radiopharmaceutical nanoparticles range in size from $10 \mathrm{~nm}$ to $1000 \mathrm{~nm}$ and are clinically approved for use as imaging agents.

However, numerous other radiopharmaceuticals have also been reported for this purpose which include 99mTc-labeled dextran, 99mTc hydroxy ethyl starch, 99mTc human serum albumin (HSA) and several labeled colloids, including gold-198-colloid, 99mTc stannous phytate, 99mTc antimony trisulfide colloid, 99mTc rhenium colloid and 99mTc colloidal albumin. However, none of these entirely fulfill the desired criteria of an ideal imaging agent. The search for an optimal nanoparticulate radiopharmaceutical is still in progress. The most essential requirement for SLN detection is that, the developed colloids should 
move to the SLN with minimal further migration. However most of these nanoparticulate systems are not ideal agents for the detection of the SLN, due to the retention of the majority of the injected dose at the peritumoral site of injection.

Studies in animals have demonstrated a very low clearance with less than $5 \%$ of the injected dose being cleared from the site of injection within the first $60 \mathrm{~min}$ and less than $2 \%$ of the injected dose accumulated in the SLN at $60 \mathrm{~min}$. The resulting low intensity of the $99 \mathrm{mTc}$ activity frequently makes the SLN localization difficult, either by imaging or with a handheld detector probe used in surgery. Filtered particles have been used for greatermovement although there is still a debate as to whether filtered particles are better than standard particles due to the possible loss of imaging agents during filtration.

The retention of nanoparticles in the first lymph node is mainly due to phagocytosis of the nanoparticles by macrophages. The total retention in the sentinel node is higher than the retention in the subsequent draining lymph nodes, since only a very small fraction of nanoparticles encountered in various studies led to the introduction of radioactive technetium-99m-sulfur colloid particles (99mTc-SC) particles as yet another method to localize the SLN in addition to the blue dye being conventionally used by the surgeons. The use of $99 \mathrm{mTc}-\mathrm{SC}$, in addition to blue dye resulted in a significant improvement in the quality of image over, that obtained by the use of blue dye alone. The blue dye was not well retained in the SLN and moved very rapidly, thus creating a difficulty for the surgeons to distinguish the sentinel node from the secondary lymph nodes. This problem was solved by the additional use of $99 \mathrm{mTc}$-SC which proved to be a better marker of the sentinel node. Although the blue dye provided the desired visual guide for the surgeon, the inclusion of $99 \mathrm{mTc}-\mathrm{SC}$ provided the desired verification that the correct lymph node was biopsied. As the imaging could be performed in the operating room prior to and during the surgery, it frequently led to a smaller operative incision and decreased the time span required for locating the sentinel node. The use of radiolabeled nanocolloids along with the blue dye thus proved to be complementary imaging agents which performed the best when used simultaneously.

\subsubsection{Magnetic nanoparticles}

The application of magnetic nanoparticles in combination with the other imaging modalities is gaining a major momentum in diverse areas of biology and medicine. Unlike many of the newer probes used in the field molecular imaging, the magnetic nanoparticles are chemically similar to a large number ofmaterials with a long history of clinical use.

Magnetic nanoparticles consist of a core of superparamagnetic iron oxide (usuallymagnetite, Fe3O4, ormaghemite, $\gamma$-Fe2O3) and are coated with a biocompatible polymer, and to this basic design, biomolecules are attached. Polymer-coated iron oxides have been used in the treatment of iron anemias since the 1960s.

Magnetic nanoparticles can be broadly categorized into two basic types namely the polymer coated and the molecularly targeted. Polymer-coated magnetic nanoparticles are those that are not designed to be recognized by a specific biomolecule (e.g. receptor, antigen). "Molecularly targeted" magnetic nanoparticles are those that are designed to bind to a specific target, typically by the attachment of a biomolecule (antibody, peptide, polysaccharide). Dextran-coated magnetic nanoparticles (SPIOs) were first introduced for hepatic imaging. Following the intravenous injection, these particles have been reported to be rapidly taken up by the liver Kupffer cells and appear hypointense or black on the MR 
images. Subsequently ultra-small magnetic nanoparticles (often referred as USPIOs) were developed, that displayed a longer blood half-life, and were taken up by the macrophages including those in lymph nodes.

Superparamagnetic particles (USPIOs) find preferential application in SLN imaging, due to the fact that they behave non-magnetically when they are not under the influence of an external magnetic field, thus preventing undesired magnetic agglomeration. To further assist in preventing agglomerations, to optimize bio-interactions with the host environment and to maximize biocompatibility, the choice of appropriate surface chemistries and functionalizations is also important. Different surface modifications have been reported to accommodate these characteristics in to the magnetic nanoparticles. These include tuning the particle size in the size range 3-30 $\mathrm{nm}$ with the particles displaying an ellipsoidal shape. A comprehensive characterization of such particles has been reported by Jolivet et al. USPIO enhanced magnetic resonance imaging (MRI) is now being used as a potential biomarker for the diagnosis of lymph node metastases. USPIOs are transported into the interstitial space and reach the SLN via the lymphatic circulation, acting as 'negative imaging' agents due to T2- and T2-weighted sequences, which can potentially identify the metastases independent of the lymph node size. However, reports also indicate that this predominant effect of USPIOs on the T2 relaxation time does not prevent the use of their properties for the T1 relaxation time when appropriate imaging sequences are chosen [150154]. USPIO nanoparticles are disseminated to the lymph nodes by two discrete pathways: a) USPIOs move into the medullary sinuses of the lymph node through venules via direct transcapillary passage, followed by phagocytosis by macrophages, b) USPIOs move into the interstitial space in the body through endothelial transcytosis, followed by uptake of the nanoparticles by draining lymphatic vessels and transport to the lymph nodes via afferent lymphatic channels. USPIOs with minor macrophage uptake and prolonged blood half-life have been shown to be useful for metastatic lymph node imaging.

Various studies have shown that USPIO particles taken up by the macrophages are transported to the interstitial spaces and subsequently to the lymph node via the lymph vessels. Thus healthy lymph node tissues produce a dark signal with a T2/T2 sequence since they are rich in macrophages which phagocytose the USPIOs. However, no such contrast modification is observed in the metastatic lymph node tissue which lacks the presence of macrophages, thus facilitating the effective imaging of this tissue. Additionally, the prolonged blood half-life of USPIOs allows their progressive access to the lymph nodes thus helping in further enhancement of the quality of image which can be procured.

Nodal disease is a self-determining adverse prognostic aspect in many types of cancers. By and far, measurement of the node size by means of imaging is the only widely established method for assessing this nodal involvement. In this regards, the superparamagnetic agents have raised immense attention because normal nodes posses high affinity towards these agents following intravenous or subcutaneous injection. The first agent, and also the most explored one is ferumoxtran-10. Numerous studies on ferumoxtran-10 have demonstrated its efficacy in metastatic lymph node imaging in various types of cancer with significant results being reported especially in case of breast cancer.

Early results with ferumoxtran-10 MRI in breast cancer showed a sensitivity of $78 \%$, a specificity of $96 \%$ and a negative predictive value of $97 \%$. The SLN procedure in breast cancer has a $3 \%-10 \%$ false negative rate; furthermore, positive internal mammary lymph nodes are missed in $17 \%$. Finally, the SLN is the only positive node in $61 \%$ lymph node positive patients. These patients all undergo axillary dissection, with subsequent high rate of 
clinically significant lymph edema. The high negative predictive value in patients with a negative ferumoxtran-10 MRI thus exhibits a possibility that axillary dissection may potentially be avoided [163]. Further studies are required to confirm the potential use of these nanoparticles in SLN localization.

Apart from the above discussed nanoparticulate systems, a variety of studies have been reported for SLN localization with various other types of magnetic nanoparticles. In one of the studies, SLN mapping of the stomach cancer has been reported using fluorescent magnetic nanoparticles (FMNP). Here the use of these nanoparticles was found to overcome problems such as unpredictable lymphatic drainage patterns and skipmetastases which are of common occurrences in these cancers and which limit the available imaging techniques for their precise diagnosis. Biocompatible silica-overcoated magnetic nanoparticles containing rhodamine B isothiocyanate (RITC) within a silica shell of controllable thickness of about $60 \mathrm{~nm}$ have also been used in some of the studies.

In another study, Surguladze et al. have reported novel magnetic nanoparticles, UNIMAG, for SLN imaging. UNIMAG represents a magnet-sensitive stable suspension of magnetite nanoparticles (magnetic fluid). After the peritumoral injection of UNIMAG, the magnetite nanoparticles have been proposed to be absorbed by the macrophages, which deliver themthrough the lymphatic capillaries to regional lymph nodes. This mechanism of magnetite nanoparticle transport is responsible for the SLN detection. As compared to the other dyestuff agents used in this direction, magnetite nanoparticles were found to actively fill the lymphatic nodes and colored them in bold black. This significantly facilitated the atraumatic separation of the lymph nodes during lymphadenectomy. Besides the intraoperative indication of lymphatic nodes, UNIMAG also provided the possibility of Xray and ultrasound imaging of SLNs and the possibility of performing their biopsy in the preoperative period. Despite these promising results, there are several problems associated with the use of magnetic nanoparticles. These limitations include (i) the possibility of embolization of the blood vessels in the target region due to accumulation of the magnetic carriers, (ii) difficulties in scaling up from animal models due to the larger distances between the target site and the magnet, and (iii) toxic responses to the magnetic carriers. However, recent pre-clinical and experimental results indicate that there is still a possibility of overcoming these limitations and use the magnetic nanoparticles to provide enhanced imaging simultaneously handling the safety issues.

\subsection{Ultrasound nanobubbles}

Particulate agents are important for the regional lymph node contrast enhancement following their subcutaneous administration.

These agents appear to traverse the endothelium of the peripheral lymphatics with subsequent localization in regional draining lymph nodes. Like other particulate nanocarriers, nanobubbles have been attempted for the ultrasound contrast enhancement of the regional lymph nodes. Nanobubble is a novel nanocarrier currently being widely investigated for the localization of the sentinel lymph node.

Nanobubbles combine properties of polymeric drug carriers, ultrasound imaging imaging agents, and enhancers of ultrasound mediated drug delivery. In biological fluids and tissues, nanobubbles are efficient reflectors of sound. Ultrasound is the most common biomedical imaging modality and ultrasound imaging agents are used to increase the reflectivity or backscatter of blood and tissues. 
Nanobubbles either comprise perfluorocarbon nanodroplets stabilized by walls made of biodegradable block copolymers or bilayered shell of albumin and an inner layer of a biodegradable polymer known as polycaprolactone. This shell encapsulates a gas such as nitrogen. Following their subcutaneous injection, these nanobubbles are tracked using ultrasound imaging.

In a study carried out in dogs for comparing the activity of microbubbles with that of submicron nanobubbles, the nanobubbles were found to bemore effective in detecting the sentinel nodes. In this study these nanobubbles were injected subcutaneously into normal dogs to target the cervical and popliteal lymph nodes. First-order (sentinel) lymph nodes and second-order sublumbar nodes were imaged intermittently from $0 \mathrm{~h}$ to at least $2 \mathrm{~h}$ following the contrast injection using continuous power Doppler mode. To confirm the lymphatic drainage patterns and sentinel lymph nodes of nanobubbles, lymphoscintigraphy studies were performed in dogs. Approximately 94\% (30 of the 32) sentinel nodes were detected during this study.

This nanobubble technology could serve as an alternative method for detecting the sentinel lymph node. This approach is also unique in that the use of ultrasound to detect the bubbles also causes the bubbles to break apart and formsmaller bubbles. It has been proposed that jets released from these nanobubbles following exposure to high-frequency ultrasound could be used to nanoinject drugs into cells. As these nanobubbles can also carry drugs, they could be used to deliver high levels of drugs to lymph nodes which could be rapidly released following insonation. However, concerns have been expressed that the energy released during this procedure lead to thermal tissue damage and thus harmful bioeffects and further investigations to verify these possible problems, are required in order to facilitate the introduction of a safe imaging modality.

\section{References}

Pinheiro, L.G.P. ; Oliveira Filho, R.S. ; Vasques, P.H.D. ; Filgueira, P.H.O. ; Aragão, D.H.P.; Barbosa, P. M. E. ; Beserra, H.E.O. ; Cavalcante, R.V. Hemosiderin: a new marker for sentinel lymph node identification. Acta Cirurgica Brasileira (Impresso), v. 24, p. 432/06, 2009.

Holland JF, Frei E, Kufe DW, Bast RC. Principles of Medical Oncology $6^{\text {th }}$ ed. Philadelphia: Saunders, 2001.

Parkin DM, Bray FI, Devesa SS. Cancer burden in the year 2000. The global picture. Eur J Cancer. 2001; 37:64-66.

Instituto Nacional do Câncer. Estimativas da incidência e mortalidade por câncer no Brasil em 2008. [cited 2009 April 12]. Available from:

URL: http://www.inca.gov.br/estimativa/2008/index.asp

Moore MP, Kinne DW. Is axillary lymph node dissection necessary in the routine management of breast cancer? Yes. Important Adv Oncol. 1996; 12:245-50.

Morton DL, Ollila DW. Critical review of the sentinel node hypothesis. Surgery. 1999; 126:815-9.

Cabanas RM. An approach for the treatment of penile carcinoma. Cancer. 1977; 39(2):456-66.

Veronesi U, Paganelli G, Niale G, Galimberti V, Luini A, Zurrida S, et al. Sentinel lymph node biopsy and axillary dissection in breast cancer: results in a large series. J Natl Cancer Inst. 1999; 91:368-73. 
Morton DL, Wen DR, Wong JH, Economou JS, Cagle LA, Storm FK, et al. Technical details of intraoperative lymphatic mapping for early stage melanoma. Arch Surg. 1992; 127:392-9.

Giuliano AE, Kirgan DM, Guenther JM, Morton DL. Lymphatic mapping and sentinel lymphadenectomy for breast cancer. Ann Surg. 1994; 220:391-401.

Krag DN, Weaver DL, Alex JC, Fairbank JT. Surgical resection and radiolocalization of the sentinel lymph node in breast cancer using a gamma probe. Surg Oncol. 1993; 2:335-9.

Pinheiro LGP, Moraes MO, Soares AH, Lopes AJT, Naguére MASP, Gondim FAL, et al. Estudo Experimental de Linfonodo sentinela na mama da cadela com azul patente e tecnécio 99. Acta Cirurgica Brasileira. 2003; 18(6):545-552.

Mertes PM, Malinovsky JM, Mouton-Faivre C, Bonnet-Boyer MC, Benhaijoub A, Lavaud F, et al. Anaphylaxis to dyes during the perioperative period: reports of 14 clinical cases. J Allergy Clin Immunol. 2008; 122:348-52.

Salhab M, Al Sarakbi W, Mokbel K. Skin and fat necrosis of the breast following methylene blue dye injection for sentinel node biopsy in a patient with breast cancer. Int Semin Surg Oncol. 2005; 2:26.

Sleth JC. Un accident anaphylactoïde imputé au bleu patenté. Faut-il changer de colorant? Ann Fr Anesth Reanim. 2008; 27:515.

Mulan MH, Deacock SJ, Quiney NF, Kissin MW. Anaphylaxis to patent blue dye during sentinel lymph node biopsy for breast cancer. Eur J Surg Oncol. 2001; 27: 218-9.

Scherer K, Studer W, Figueiredo V, Bircher AJ. Anaphylaxis to isosulfan blue and crossreactivity to patent blue $\mathrm{V}$ : case report and review of the nomenclature of vital blue dyes. Ann Allergy Asthma Immunol. 2006; 96: 497-500.

Wohrl S, Focke M, Hinterhuber G, Stingl G, Binder M. Near-Fatal Anaphylaxis to Patent Blue V. Br J Dermatol. 2004; 150:1037-8.

M. Hamoudeh, M.A. Kamleh, R. Diab, H. Fessi, Radionuclides delivery systems for nuclear imaging and radiotherapy of cancer, Adv. Drug Deliv. Rev. 60 (2008) 1329-1346.

A.D. Bangham, M.M. Standish, J.C. Watkins, Diffusion of univalent ions across the lamellae of swollen phospholipids, J. Mol. Biol. 13 (1965) 238-252.

H.E.Wang,H.M. Yu, Y.C. Lu,N.N.Heish, Y.L. Tseng, K.L.Huang, K.T. Chuang, C.H. Chen, J.J. Hwang,W.J. Lin, S.J.Wang, G. Ting, P.J. Whang,W.P. Deng, Internal radiotherapy and dosimetric study for 111In/177Lu-pegylated liposomes conjugates in tumor bearing mice, Nucl. Instrum. Methods, A 569 (2) (2006) 533537.

W.T. Phillips, R. Klipper, B. Goins, Use of $(99 \mathrm{~m})$ Tc-labeled liposomes encapsulating blue dye for identification of the sentinel lymph node, Nucl. Med. 42 (2001) 446-451.

T.A. Elbayoumi, V.P. Torchilin, Enhanced accumulation of long-circulating liposomes modified with the nucleosome-specific monoclonal antibody $2 \mathrm{C} 5$ in various tumours in mice: gamma-imaging studies, Eur. J. Nucl.Med.Mol. Imaging 33 (2006) 1196-1205.

S. Dagar, A. Krishnadasa, I. Rubinstein,M.J. Blend, H. Önyüksel, VIP grafted sterically stabilized liposomes for targeted imaging of breast cancer: in vivo studies, J. Control. Release 91 (2003) 123-133.

V.P. Torchilin, Surface-modified liposomes in $\gamma$ and MR imaging, Adv. Drug Deliv. Rev. 24 (1997) 301-313. 
A.W. Segal, G. Gregoriadis, C.D. Black, Liposomes as vehicles for the local release of drugs, Clin. Sci. Mol. Med. 49 (1975) 99-106.

M.P. Osborne, V.J. Richardson, K. Jeyasingh, B.E. Ryman, Radionuclide-labelled liposomes - a newlymphnode imaging agent, Int. J.Nucl.Med.Biol. 6 (1979) 75-83.

H.M. Patel, K.M. Boodle, R. Vaughan-Jones, Assessment of the potential uses of liposomes for lymphoscintigraphy and lymphatic drug delivery. Failure of $99 \mathrm{~m}$ - technetiumto represent intact liposomes in lymph nodes, Biochim. Biophys. Acta 801 (1984) 7686.

G.M. Barratt, N.S. Tuzel, B.E. Ryman, The labeling of liposomal membranes with radioactive technetium, in: G. Gregoriadis (Ed.), Liposome Technology, vol. 2, CRC Press, Boca Raton, FL, 1984, pp. 94-105.

P. Laverman, E. Dams,W. Oyen, G. Storm, E. Koenders, R. Prevost, J. van derMeer, F. Corstens, O. Boerman, A novel method to label liposomes with $99 \mathrm{mTc}$ by the hydrazino nicotinyl derivative, J. Nucl. Med. 40 (1999) 192-197.

C. Tilcock, Q.F. Ahkong, D. Fisher, 99mTc-labeling of lipid vesicles containing the lipophilic chelator PE-DTPA: effect of tin-to chelate ratio, chelate content and surface polymer on labeling efficiency and biodistribution behavior, Nucl. Med. Biol. 21 (1994) 8996.

P. Hirnle, R. Harzmann, J.K. Wright, Patent blue V encapsulation in liposomes: potential applicability to endolympatic therapy and preoperative chromolym phography, Lymphology 21 (1988) 187-189.

B. Pump, P. Hirnle, Preoperative lymph-node staining with liposomes containing patent blue violet. A clinical case report, J. Pharm. Pharmacol. 48 (1996) 699-701.

W.T. Phillips, R. Klipper, B. Goins, Novel method of greatly enhanced delivery of liposomes to lymph nodes, Pharmacol. Exp. Ther. 295 (2000) 309-313.

L.A.Medina, S.M. Calixto, R. Klipper,W.T. Phillips, B. Goins, Avidin/biotinliposome system injected in the pleural space for drug delivery to mediastinal lymph nodes, J. Pharm. Sci. 93 (2004) 2595-2608.

L.A. Medina, R. Klipper,W.T. Phillips, B. Goins, Pharmacokinetics and biodistribution of [111ln]-avidin and [99mTc]-biotin-liposomes injected in the pleural space for the targeting of mediastinal nodes, Nucl. Med. Biol. 31 (2004) 41-51.

E.M. Plut, G.H. Hinkle, W. Guo, R.J. Lee, Kit formulation for the preparation of radioactive blue liposomes for sentinel node lymphoscintigraphy, J. Pharm. Sci. 91 (2002) 17171732.

M. Dieter, R. Schubert, P. Hirnle, Blue liposomes for identification of the sentinel lymph nodes in pigs, Lymphology 36 (2003) 39-47.

V.P. Torchilin, Recent advances with liposomes as pharmaceutical carriers, Nat. Rev., Drug Discov. 4 (2005) 145-160.

S. Stiriba, H. Frey, R. Haag, Dendritic polymers in biomedical applications: from potential to clinical use in diagnostics and therapy, Angew. Chem., Int. Ed. Engl. 41 (2002) 1329-1334.

D.A. Tomalia, H. Baker, J.R. Dewald,M. Hall, G. Kallos, S.Martin, J. Roeck, J. Ryder, P. Smith, A new class of polymers: starburst-dendritic macromolecules, Polym. J. 17 (1985) 117-132.

V.J. Venditto, C.S. Regino, M.W. Brechbiel, PAMAM dendrimer based macro molecules as improved contrast agents, Mol. Pharmaceutics 2 (2005) 302-311. 
J.M. Fréchet, M. Henni, I. Gitsov, S. Aoshima, M.R. Leduc, R.B. Grubbs, Self-condensing vinyl polymerization: an approach to dendritic materials, Science 269 (1995) 10801083.

R. Esfand, D.A. Tomalia, Poly (amidoamine) (PAMAM) dendrimers: from biome 1351 micry to drug delivery and biomedical applications, Drug Discov. Today 6 (2001) 1352 427-436.

V.S. Talanov, C.A. Regino, H. Kobayashi, M. Bernardo, P.L. Choyke, M.W. Brechbiel, Dendrimer-based nanoprobe for dual modality magnetic resonance and fluorescence imaging, Nano Lett. 6 (2006) 1459-1463.

E.C.Wiener,M.W. Brechbiel, H. Brothers, R.L.Magin, O.A. Gansow, D.A. Tomalia, P.C. Lauterbur, Dendrimer-based metal chelates: a new class of magnetic resonance imaging contrast agents, Magn. Reson. Med. 31 (1994) 1-8.

R.H.E. Hudson, M. Damha, Nucleic acid dendrimers: novel biopolymer structures, J. Am. Chem. Soc. 115 (1993) 2119-2124.

Y. Koyama, V.S. Talanov, M. Bernardo, Y. Hama, C.A.S. Regino, M.W. Brechbiel, P.L. Choyke, H. Kobayashi, A dendrimer-based nanosized contrast agent dual-labeled for magnetic resonance and optical fluorescence imaging to localize the sentinel lymph node in mice, J. Magn. Reson. Imaging 25 (2007) 866-871.

H. Kobayashi, S. Kawamoto, Y. Sakai, P.L. Choyke, R.A. Star,M.W. Brechbiel, N. Sato, Y. Tagaya, J.C. Morris, T.A. Waldmann, Lymphatic drainage imaging of breast cancer in mice by micro-magnetic resonance lymphangiography using a nano size paramagnetic contrast agent, J. Natl. Cancer Inst. 96 (2004) 703-708.

H. Kobayashi, S. Kawamoto, P.L. Choyke, N. Sato, M.V. Knopp, R.A. Star, T.A. Waldmann, Y. Tagaya, M.W. Brechbiel, Comparison of dendrimer-based macromolecular contrast agents for dynamic micromagnetic resonance lymphangiography, Magn. Reson. Med. 50 (2003) 758-766.

A.P. Alivisatos, Semiconductor clusters, nanocrystals, and quantum dots, Science 271 (1996) 933-937.

M. Bruchez, M. Moronne, P. Gin, S. Weiss, A.P. Alivisatos, Semiconductor nanocrystals as fluorescent biological labs, Science 281 (1998) 2013-2016.

S. Liu, C.M. Lee, S. Wang, D.R. Lu, A new bioimaging carrier for quantum dot nanocrystals - phospholipid nanoemulsion mimicking natural lipoprotein core, Drug Deliv. 13 (2006) 159-164.

B. Dubertret, P. Skourides, D.J. Norris, V. Noireaux, A.H. Brivanlou, A. Libchaber, In vivo imaging of quantum dots encapsulated in phospholipid micelles, Science 298 (2002) 1759-1762.

X. Gao,Y. Cui, R.M. Levenson, L.W. Chung, S.Nie, Invivo cancer targeting and imaging with semiconductor quantum dots, Nat. Biotechnol. 22 (2004) 969-976.

W.C.W. Chan, S. Nie, Quantum dot bioconjugates for ultrasensitive nonisotopic detection, Science 281 (1998) 2016-2018.

W.C.W. Chan, D.J.Maxwell, X. Gao, R.E. Bailey,M. Han, S. Nie, Luminescent QDs for multiplexed biological detection and imaging, Curr. Opin. Biotechnol. 13 (2002) 4046.

M. Han, X. Gao, J.Z. Su, S. Nie, Quantum dot-tagged microbeads for multiplexed optical coding of biomolecules, Nat. Biotechnol. 19 (2001) 631-635. 
C.M. Niemeyer, Nanoparticles, proteins, and nucleic acids: biotechnology meets materials science, Angew. Chem., Int. Ed. Engl. 40 (2001) 4128-4158.

C.A. Leatherdale, W.K. Woo, F.V. Mikulec, M.G. Bawendi, On the absorption cross section of CdSe nanocrystal quantum dots, J. Phys. Chem. Biol. 106 (2002) 7619-7622.

B. Ballou, Quantumdot surfaces for use in vivo and in vitro, Curr. Top. Dev. Biol. 70 (2005) 103-120.

B. Ballou, B.C. Lagerholm, L.A. Ernst, M.P. Bruchez, A.S. Waggoner, Noninvasive imaging of quantum dots in mice, Bioconjug. Chem. 15 (2004) 79-86.

S. Kim, Y.T. Lim, E.G. Soltesz, A.M. De Grand, J. Lee, A. Nakayama, J.A. Parker, T. Mihaljevic, R.G. Laurence, D.M. Dor, L.H. Cohn,M.G. Bawendi, J.V. Frangioni, Near infrared fluorescent type II quantum dots for sentinel lymph node mapping, Nat. Biotechnol. 22 (2004) 93-97.

B. Ballou, L.A. Ernst, A.S.Waggoner, Fluorescence imaging of tumors in vivo, Curr. Med. Chem. 12 (2005) 795-805.

J.V. Frangioni, In vivo near-infrared fluorescence imaging, Curr. Opin. Chem. Biol. 7 (2003) 626-634.

Y.T. Lim, S. Kim, A. Nakayama,N.E. Stott,M.G. Bawendi, J.V. Frangioni, Selection of QD wavelengths for biomedical assays and imaging, Mol. Imaging 2 (2003) 50-64.

A. Nakayama, F. Del Monte, R.J. Hajjar, J.V. Frangioni, Functional near-infrared fluorescence imaging for cardiac surgery and targeted gene therapy,Mol. Imaging 1 (2002) 365-377.

A.M. De Grand, J.V. Frangioni, An operational near-infrared fluorescence imaging system prototype for large animal surgery, Technol. Cancer Res. Treat. 2 (2003) 553-562.

X.Michalet, F.F. Pinaud, L.A. Bentolila, J.M. Tsay, S. Doose, J.J. Li, G. Sundaresan, A.M. Wu, S.S. Gambhir, S. Weiss, Quantum dots for live cells, in vivo imaging, and diagnostics, Science 307 (2005) 538-544.

S. Kim, J.P. Zimmer, S. Ohnishi, J.B. Tracy, J.V. Frangioni,M.G. Bawendi, Engineering InAsxP1-x/InP/ZnSe III-V alloyed core/shell quantum dots for the near-infrared, J. Am. Chem. Soc. 127 (2005) 10526-10532.

B. Ballou, L.A. Ernst, S. Andreko, T. Harper, J.A.J. Fitzpatrick, A.S. Waggoner, M.P. Bruchez, Sentinel lymph node imaging using quantum dots in mouse tumor models, Bioconjug. Chem. 18 (2007) 389-396.

N.V. Gopee, D.W. Roberts, P.Webb, C.R. Cozart, P.H. Siitonen, A.R.Warbritton,W.W. Yu, V.L. Colvin, N.J. Walker, P.C. Howard, Migration of intradermally injected quantum dots to sentinel organs in mice, Toxicol. Sci. 98 (2007) 249-257.

A.M. Derfus, W.C.W. Chan, S.N. Bhatia, Probing the cytotoxicity of semiconductor quantum dots, Nano Lett. 4 (2004) 11-18.

R. Hardman, A toxicologic review of quantum dots: toxicity depends on physicochemical and environmental factors, Environ. Health Perspect. 114 (2006) 165-172.

A. Hoshino, K. Fujioka, T. Oku,M. Suga, Y.F. Sasaki, T. Ohta,M. Yasuhara, K. Suzuki, K. Yamamoto, Physicochemical properties and cellular toxicity of nanocrystal quantum dots depend on their surface modification, Nano Lett. 4 (2004) 2163-2169.

R. Jain et al. / Journal of Controlled Release 138 (2009) 90-102[120] C. Kirchner, T. Liedl, S. Kudera, T. Pellegrino, A. Munoz Javier, H.E. Gaub, S. Stolzle, N. Fertig, W.J. Parak, Cytotoxicity of colloidal CdSe and CdSe/ZnS nanoparticles, Nano Lett. 5 (2005) 331-338. 
S.M. Moghimi, A.E. Hawley, N.M. Christy, T. Gray, L. Illum, S.S. Davis, Surface engineered nanospheres with enhanced drainage into lymphatics and uptake by macrophages of the regional lymph nodes, FEBS Lett. 344 (1994) 25-30.

K.A. Hultborn, L.G. Larsonn, I. Ragnhult, The lymph drainage from the breast to the axillary and parasternal lymph nodes studied with the aid of colloidal Au 198, Acta. Radiol. 43 (1955) 52-63.

J. Weinberg, E.M. Greaney, Identification of regional lymph nodes by means of vital staining dye during surgery of gastric cancer, Surg. Gynecol. Obstet. 90 (1950) 561-567.

D.L.Morton, D.R.Wen, J.H.Wong, J.S. Economou, L.A. Cagle, F.K. Storm, L.J. Foshag, A.J. Cochran, Technical details of intraoperative lymphatic mapping for early stage melanoma, Arch. Surg. 127 (1992) 392-399.

J.C. Alex, D.N. Krag, Gamma probe guided localization of lymph nodes, Surg. Oncol. 2 (1993) 137-143.

A.J. Wilhelm, G.S. Mijnhout, E.J. Franssen, Radiopharmaceuticals in sentinel lymph-node detection - an overview, Eur. J. Nucl. Med. 26 (1999) S36-42.

J.C. Hung, G.A. Wiseman, H.W. Wahner, B.P. Mullan, T.R. Taggart, W.L. Dunn, Filtered technetium-99m-sulfur colloid evaluated for lymphoscintigraphy, J. Nucl. Med. 36 (1995) 1895-1901.

R.C.Martin 2nd,M.J. Edwards, S.L.Wong, T.M. Tuttle, D.J. Carlson, C.M. Brown, R.D. Noyes, R.L. Glaser, D.J. Vennekotter, P.S. Turk, P.S. Tate, A. Sardi, P.B. Cerrito, K.M. McMasters, University of Louisville breast cancer sentinel lymph node study, practical guidelines for optimal gamma probe detection of sentinel lymph nodes in breast cancer: results of a multi-institutional study, Surgery 128 (2000) 139-144.

C. Oussoren, G. Storm, Role of macrophages in the localisation of liposomes in lymph nodes after subcutaneous administration, Int. J. Pharm. 183 (1999) 37-41.

S.L. Focht, Lymphatic mapping and sentinel lymph node biopsy, AORN J. 69 (1999) $802-$ 809.

A. Gradilone, D. Ribuffo, I. Silvestri, E. Cigna, P. Gazzaniga, I. Nofroni, G. Zamolo, L. Frati, N. Scuderi, A.M. Aglianò, Detection of melanoma cells in sentinel lymph nodes by reverse transcriptase-polymerase chain reaction: prognostic significance, Ann. Surg. Oncol. 11 (2004) 983-987.

J.H.Wong, L.A. Cagle, D.L.Morton, Lymphatic drainage of skin to a sentinel lymph node in a feline model, Ann. Surg. 214 (1991) 637-641.

H.S. Cody III, J. Fey, T. Akhurst, M. Fazzari, M. Mazumdar, H. Yeung, S.D.J. Yeh, P.I. Borgen, Complementarity of blue dye and isotope in sentinel node localization for breast cancer: univariate and multivariate analysis of 966 procedures, Ann. Surg. Oncol. 8 (2001) 13-19.

D.L. Burns, E.A. Mascioli, B.R. Bistrian, Parenteral iron dextran therapy: a review, Nutrition 11 (1995) 163-168.

S.T. Callender, Treatment of iron deficiency, Clin. Haematol. 11 (1982) 327-338.

L. Josephson, Magnetic nanoparticles for MR imaging, in: M. Ferrari (Ed.), BioMEMS and Biomedical Nanotechnology, vol. 1, Springer Science, New York, 2006, pp. 227-228.

A. Moore, R. Weissleder, A. Bogdanov Jr., Uptake of dextran-coated monocrystalline iron oxides in tumor cells and macrophages, J. Magn. Reson. Imaging 7 (6) (1997) 11401145. 
D.D. Stark, R.Weissleder,G. Elizondo, P.F.Hahn, S. Saini, L.E. Todd, J.Wittenberg, J.T. Ferrucci, Superparamagnetic iron oxide: clinical application as a contrast agent for MR imaging of the liver, Radiology 168 (1988) 297-301.

R. Weissleder, G. Elizondo, J. Wittenberg, A.S. Lee, L. Josephson, T.J. Brady, Ultrasmall superparamagnetic iron oxide: an intravenous contrast agent for assessing lymph nodes with MR imaging, Radiology 175 (1990) 494-498.

M.G. Harisinghani, J. Barentsz, P.F. Hahn, W.M. Deserno, S. Tabatabaei, C.H. van de Kaa, J. de la Rosette, R.Weissleder, Noninvasive detection of clinically occult lymph node metastases in prostate cancer, N. Engl. J. Med. 348 (2003) 2491-2499.

R.E. Rosensweig, Magnetic fluids, Sci. Am. 247 (1982) 136-145.

H. Itoh, T. Sugimoto, Systematic control of size, shape, structure, and magnetic properties of uniform magnetite and maghemite particles, Colloid Interface Sci. 265 (2002) 283295.

O. Bomati-Miguel, M.P. Morales, P. Tartaj, J. Ruiz-Cabello, P. Bonville,M. Santos, X.Q. Zhao, S. Veintemillas-Verdaguer, Fe-based nanoparticulate metallic alloys as contrast agents for magnetic resonance imaging, Biomaterials 26 (2005) 5695-5703.

R. Vijayakumar, Y. Koltypin, I. Felner, A. Gedanken, Sonochemical synthesis and characterization of pure nanometer-sized Fe304 particles, Mater. Sci. Eng., A 286 (2000) 101-105.

R.M. Cornell, U. Schwertmann, The Iron Oxides. Structure, Properties, Reactions, Occurrence and Uses, VCH, Weinheim, Germany, 1996, pp. 344-345.

E. Tronc, J.P. Jolivet, Surface effects on magnetically coupled gamma-Fe2O3 colloids, Hyperfine Interact. 28 (1986) 525-528.

E. Tronc, D. Fiorani, M. Nogues, A.M. Testa, F. Lucari, F. D'Orazio, J.M. Greneche,W. Wernsdorfer, N. Galvez, C. Chaneac, D. Mailly, J.P. Jolivet, Surface effects in noninteracting and interacting y-Fe2O3 nanoparticles, J. Magn. Magn. Mater. 262 (2003) 6-14.

E.D. Parker, V. Dupuis, F. Ladieu, J.P. Bouchaud, E. Dubois, R. Perzynski, E. Vincent, Spinglass behavior in an interacting $\gamma$-Fe2O3 nanoparticle system, Phys. Rev., B 77 (2008) 104428.

J.L. Dormann, L. Dormann, L. Spinu, E. Tronc, J.P. Jolivet, F. Lucari, F. D'Orazio, D. Fiorani, Effect of interparticle interactions on the dynamical properties of y-Fe203 nanoparticles, J. Magn.Magn. Mater. 183 (1998) L255-L260.

S. Saini, D.D. Stark, P.F. Hahn, J. Wittenberg, T.J. Brady, J.T. Ferrucci Jr., Ferrite particles: a superparamagnetic MR contrast agent for the reticulo endothelial system, Radiology 162 (1987) 211-216.

A. Hemmingsson, J. Carlsten, A. Ericsson, J. Klaveness, G.O. Sperber, K.A. Thuomas, Relaxation enhancement of the dog liver and spleen by biodegradable superparamagnetic particles in proton magnetic resonance imaging, Acta. Radiol. 28 (1987) 703-705.

J.T. Ferrucci, D.D. Stark, Iron oxide-enhanced MR imaging of the liver and spleen: review of the first 5 years, AJR Am. J. Roentgenol. 155 (1990) 943-950.

A. Chachuat, B. Bonnemain, European clinical experience with Endorem. A new contrast agent for liver MRI in 1000 patients, Radiologe 35 (1995) S274-S276.

P. Reimer, B. Tombach, Hepatic MRI with SPIO: detection and characterization of focal liver lesions, Eur. Radiol. 8 (1998) 1198-1204. 
C. Corot, P. Robert, J.M. Idee, M. Port, Recent advances in iron oxide nanocrystal technology for medical imaging, Adv. Drug Deliv. Rev. 58 (2006) 1471-1504.

M. Ferrari, Cancer nanotechnology: opportunities and challenges, Nat. Rev., Cancer 5 (2005) 161-171.

Q.A. Pankhurst, J. Connolly, S.K. Jones, J. Dobson, Applications of magnetic nanoparticles in biomedicine, J. Phys., D, Appl. Phys. 36 (2003) R167-R181.

J. Dobson, Magnetic nanoparticles for drug delivery, Drug Dev. Res. 67 (2006) 55-60.

M.G. Harisinghani, J. Barentsz, P.F. Hahn, W.M. Deserno, S. Tabatabaei, C. Hulsbergen van de Kaa, J. De la Rosette, R. Weissleder, Noninvasive detection of clinically occult lymph-node metastases in prostate cancer, N. Engl. J. Med. 348 (2003) 2491-2499.

J.M. Rogers, J. Lewis, L. Josephson, Visualization of superiormesenteric lymph nodes by the combined oral and intravenous administration of the ultra-small superparamagnetic iron oxide AMI-227, Magn. Reson. Imaging 12 (1994) 11611165.

Y. Anzai, S. McLachlan, M. Morris, R. Saxton, R.B. Lufkin, Dextran-coated superparamagnetic iron oxide an MR contrast agent for assessing lymph nodes in the head and neck, Am. J. Neuroradiol. 15 (1994) 87-94.

M.F. Bellin, C. Roy, K. Kinkel, D. Thoumas, S. Zaim, D. Vanel, C. Tuchmann, F. Richard, D. Jacqumin, A. Delcourt, E. Challier, T. Lebret, P. Cluzel, Lymph node metastases: safety and effectiveness of MR imaging with ultrasmall superparamagnetic iron oxide particles - initial clinical experience, Radiology 207 (1998) 799-808.

S.C.A.Michel, T.M. Keller, J.M. Fröhlich, D. Fink, R. Caduff, B. Seifert, B.Marincek, R. KubikHuch, Preoperative breast cancer staging: MR imaging of the axilla with ultrasmall superparamagnetic iron oxide enhancement, Radiology 225 (2002) 527-536.

J.S. Kim, T.J. Yoon, H.K. Kim, S.S. Kim, H.S. Chae,M.G. Choi, Y.J. Kim, G.C. Yi, Y.S. Cho, Sentinel lymph node mapping of the stomach using fluorescent magnetic nanoparticles in rabbits, Korean J. Gastroenterol. 51 (2008) 19-24.

G.B. Surguladze, R. Zhorzoliani, T. Tskitishvili, Novel method of sentinel lymph node detection in malignant tumors using preparation 'UNIMAG', Breast Cancer Res. 9 (2007) P15.

C. Chambon, O. Clément, A. Le Blanche, E. Schouman-Claeys, G. Frija, Superparamagnetic iron oxides as positive MR contrast agents: in vitro and in vivo evidence, Magn. Reson. Imaging 11 (1993) 509-519.

E. Canet, D. Revel, R. Forrat, C. Baldy-Porcher, M. de Lorgeril, L. Sebbag, J.P. Vallee, D. Didier, M. Amiel, Superparamagnetic iron oxide particles and positive enhancement for myocardial perfusion studies assessed by subsecond T1-weighted MRI, Magn. Reson. Imaging 11 (1993) 1139-1145.

F. Forsberg, W.T. Shi, Physics of contrast microbubbles, in: B.B. Goldberg, J.S. Raichlen, F. Forsberg, Martin Dunitz (Eds.), Ultrasound Contrast Agents: Basic Principles and Clinical Applications, 2001, pp. 15-24.

P.A. Dayton, J.E. Chomas, A.F. Lum, J.S. Allen, J.R. Lindner, S.I. Simon, K.W. Ferrara, Optical and acoustical dynamics of microbubble contrast agents inside neutrophils, Biophys. J. 80 (2001) 1547-1556.

E.C. Unger, T.O. Matsunaga, T. McCreery, P. Schumann, R. Sweitzer, R. Quigley, Therapeutic applications of microbubbles, Eur. J. Radiol. 42 (2002) 160-168. 
F. Robert, M.D. Mattrey, M.D. Yuko Kono, K. Baker, T. Peterson, Sentinel lymph node imaging with microbubble ultrasound contrast material, Acad. Radiol. 9 (2002) S231-S235.

N. Rapoport, Z. Gao, A. Kennedy, Multifunctional nanoparticles for combining ultrasonic tumor imaging and targeted chemotherapy, J. Natl. Cancer Inst. 99 (2007) 10951106.

F. Yang, A. Gu, Z. Chen, N. Gu, M. Ji, Multiple emulsion microbubbles for ultrasound imaging, Mater. Lett. 62 (2008) 121-124.

Z. Gao, A.M. Kennedy, D.A. Christensen, N.Y. Rapoport, Drug-loaded nano/ microbubbles for combining ultrasonography and targeted chemotherapy, Ultrasonics 48 (2008) 260-270.

N. Rapoport, K.H. Nam, Z. Gao, D.A. Cristensen, A.M. Kennedy, Acoustic properties of multifunctional nanomicrobubbles used in ultrasonography and ultrasoundmediated chemotherapy, J. Acoust. Soc. Am. 123 (2008) 3217.

E.R. Wisner, K.W. Ferrara, R.E. Short, T.B. Ottoboni, J.D. Gabe, D. Patel, Sentinel node detection using contrast-enhanced power Doppler ultrasound lymphography, Invest. Radiol. 383 (2003) 58-65.

M. Postema, A.V.Wamel, C.T. Lancee, N.D. Jong, Ultrasound-induced encapsulated microbubble phenomena, Ultrasound Med. Biol. 30 (2004) 827-840.

P.A. Dijkmans, Microbubbles and ultrasound: from diagnosis to therapy, Eur. J. Echocardiog. 5 (2004) 245-256.

E. Stride, N. Saffari, The potential for thermal damage posed by microbubble ultrasound contrast agents, Ultrasonics 42 (2004) 907-913.

Hung WK, et al:: Randomized clinical trial comparing blue dye with combined dye and isotope for sentinel lymph node biopsy in breast cancer. Br J Surg 92: 1494-1497, 2005.

Goyal A, Douglas-Jones AG, Newcombe RG, Mansel RE: Effect of lymphatic tumor burden on sentinel lymph node biopsy in breast cancer. Breast J 11: 188-194, 2005.

Cox CE, et al.: Age and body mass index may increase the chance of failure in sentinel lymph node biopsy for women with breast cancer. Breast J 8: 88-91, 2002.

Derossis AM, Fey JV, Cody HS III, Borgen PI: Obesity influences outcome of sentinel lymph node biopsy in early-stage breast cancer. J Am Coll Surg 197: 896-901, 2003.

Birdwell RL, et al.: Breast cancer: variables affecting sentinel lymph node visualization at preoperative lymphoscintigraphy. Radiology 220: 47-53, 2001.

Goyal A, et al.: Sentinel lymph node biopsy in male breast cancer patients. Eur J Surg Oncol 30: 480-483, 2004. 


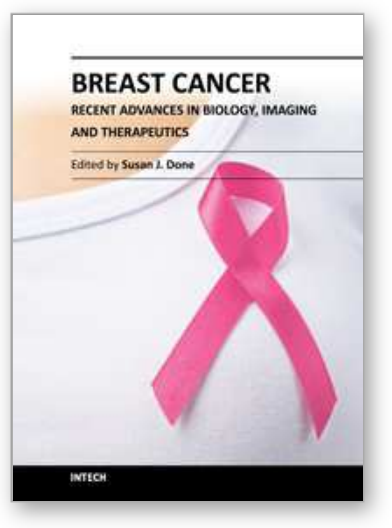

\author{
Breast Cancer - Recent Advances in Biology, Imaging and \\ Therapeutics \\ Edited by Dr. Susan Done
}

ISBN 978-953-307-730-7

Hard cover, 428 pages

Publisher InTech

Published online 14, December, 2011

Published in print edition December, 2011

In recent years it has become clear that breast cancer is not a single disease but rather that the term encompasses a number of molecularly distinct tumors arising from the epithelial cells of the breast. There is an urgent need to better understand these distinct subtypes and develop tailored diagnostic approaches and treatments appropriate to each. This book considers breast cancer from many novel and exciting perspectives. New insights into the basic biology of breast cancer are discussed together with high throughput approaches to molecular profiling. Innovative strategies for diagnosis and imaging are presented as well as emerging perspectives on breast cancer treatment. Each of the topics in this volume is addressed by respected experts in their fields and it is hoped that readers will be stimulated and challenged by the contents.

\title{
How to reference
}

In order to correctly reference this scholarly work, feel free to copy and paste the following:

L.G. Porto Pinheiro, P.H.D. Vasques, M. Maia, J.I.X. Rocha and D.S. Cruz (2011). Sentinel Lymph Node Biopsy: Actual Topics, Breast Cancer - Recent Advances in Biology, Imaging and Therapeutics, Dr. Susan Done (Ed.), ISBN: 978-953-307-730-7, InTech, Available from: http://www.intechopen.com/books/breastcancer-recent-advances-in-biology-imaging-and-therapeutics/sentinel-lymph-node-biopsy-actual-topics

\section{INTECH}

open science | open minds

\section{InTech Europe}

University Campus STeP Ri

Slavka Krautzeka 83/A

51000 Rijeka, Croatia

Phone: +385 (51) 770447

Fax: +385 (51) 686166

www.intechopen.com

\section{InTech China}

Unit 405, Office Block, Hotel Equatorial Shanghai

No.65, Yan An Road (West), Shanghai, 200040, China

中国上海市延安西路65号上海国际贵都大饭店办公楼 405 单元

Phone: +86-21-62489820

Fax: $+86-21-62489821$ 
(C) 2011 The Author(s). Licensee IntechOpen. This is an open access article distributed under the terms of the Creative Commons Attribution 3.0 License, which permits unrestricted use, distribution, and reproduction in any medium, provided the original work is properly cited. 